This item was submitted to Loughborough's Research Repository by the author.

Items in Figshare are protected by copyright, with all rights reserved, unless otherwise indicated.

\title{
Measurements and LES calculations of turbulent premixed flame propagation past repeated obstacles
}

PLEASE CITE THE PUBLISHED VERSION

http://dx.doi.org/10.1016/j.combustflame.2011.05.008

\section{PUBLISHER}

(c) The Combustion Institute. Published by Elsevier Inc.

\section{VERSION}

AM (Accepted Manuscript)

\section{LICENCE}

CC BY-NC-ND 4.0

\section{REPOSITORY RECORD}

Gubba, Sreenivasa Rao, Salah S. Ibrahim, W. Malalasekera, and Assaad R. Masri. 2019. "Measurements and LES Calculations of Turbulent Premixed Flame Propagation Past Repeated Obstacles". figshare. https://hdl.handle.net/2134/9125. 
This item was submitted to Loughborough's Institutional Repository (https://dspace.lboro.ac.uk/) by the author and is made available under the following Creative Commons Licence conditions.

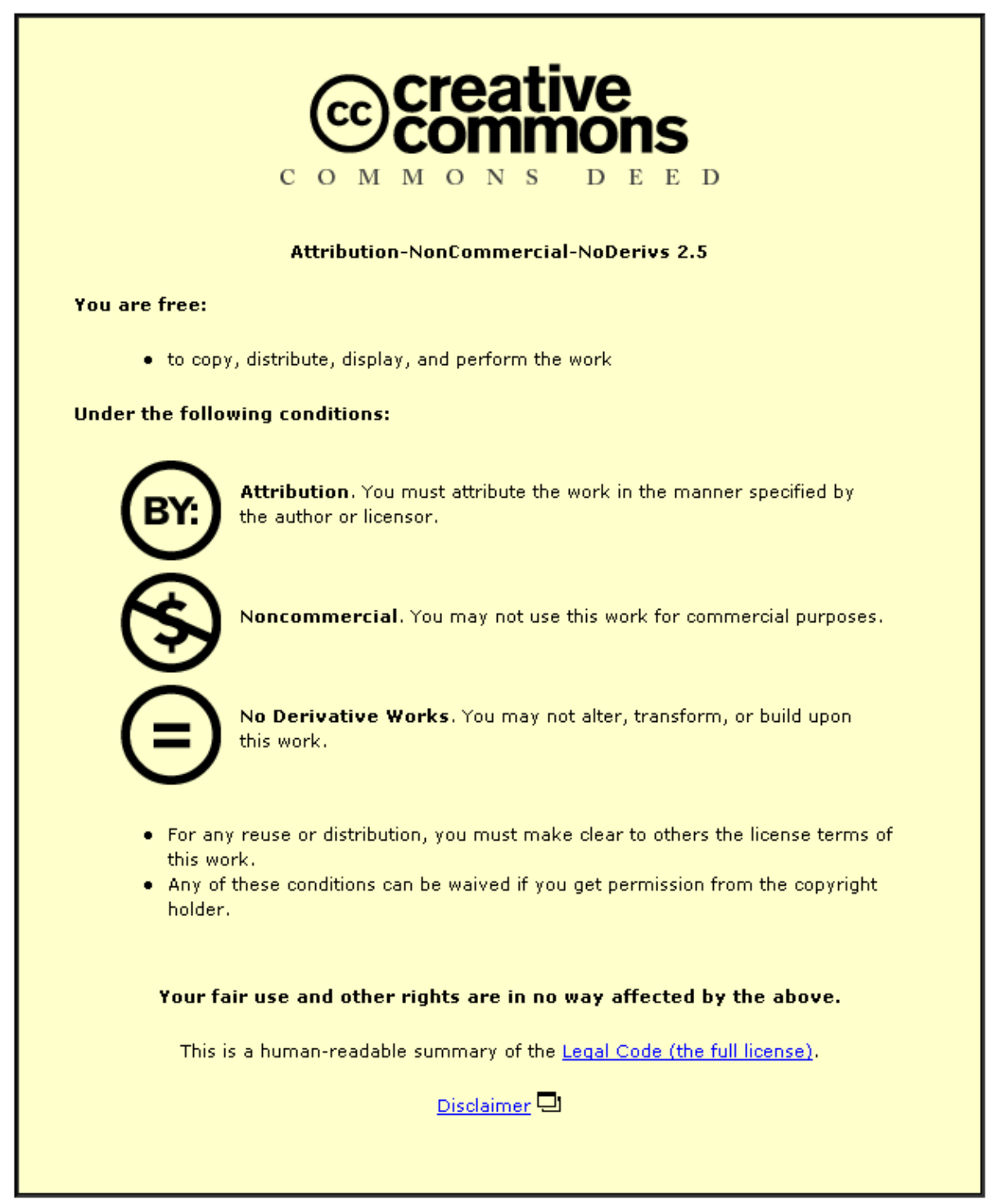

For the full text of this licence, please go to: http://creativecommons.org/licenses/by-nc-nd/2.5/ 
The citation for this article is:

Gubba, S.R., Ibrahim, S.S., Malalasekera, W., and Masri, A.R., Measurements and LES calculations of turbulent premixed flame propagation past repeated obstacles Combustion and Flame 158 (2011) 2465-2481, doi:10.1016/j.combustflame.2011.05.008

\title{
Measurements and LES Calculations of Turbulent Premixed Flame Propagation past Repeated Obstacles
}

\section{Sreenivasa Rao Gubba ${ }^{a, 1, *}$, Salah S Ibrahim ${ }^{b}$, Weertunge Malalasekera ${ }^{c}$ and Assaad R Masri $^{\mathrm{d}}$}

\author{
${ }^{\mathrm{a}}$ Wolfson School of Mechanical and Manufacturing Engineering \\ Loughborough University \\ Loughborough, LE11 3TU, UK \\ S.R.Gubba@Leeds.ac.uk
${ }^{\mathrm{b}}$ Aeronautical and Automotive Engineering
Loughborough University
Loughborough, LE11 3TU, UK
S.S.Ibrahim@lboro.ac.uk
${ }^{c}$ Wolfson School of Mechanical and Manufacturing Engineering
Loughborough University Loughborough, LE11 3TU, UK
W.Malalasekera@lboro.ac.uk
${ }^{\mathrm{d}}$ School of Aerospace, Mechanical and Mechatronic Engineering
The University of Sydney, NSW, 2006, Australia
masri@aeromech.usyd.edu.au

\footnotetext{
*Corresponding author
}

\footnotetext{
${ }^{1}$ Current Address: School of Process Environment and Materials Engineering University of Leeds, Leeds, LS2 9JT, UK

S.R.Gubba@Leeds.ac.uk

Tel: +44 (0) 1133433824 Fax: +44 (0) 1132467310 


\begin{abstract}
Measurements and large eddy simulations (LES) have been carried out for a turbulent premixed flame propagating past solid obstacles in a laboratory scale combustion chamber. The mixture used is a stoichiometric propane/air mixture, ignited from rest. A wide range of flow configurations are studied. The configurations vary in terms of the number and position of the built-in solid obstructions. The main aim of the present study is two folded. First, to validate a newly developed dynamic flame surface density (DFSD) model over a wide range of flow conditions. Second, to provide repeatable measurements of the flow and combustion in a well-controlled combustion chamber. A total of four groups are derived for qualitative and quantitative comparisons between predicted results and experimental measurements. The concept of groups offers better understanding of the flame-flow interactions and the impact of number and position of the solid baffle plates with respect to the ignition source. Results are presented and discussed for the flame structure, position, speed and accelerations at different times after ignitions. The pressure-time histories are also presented together with the regimes of combustion for all flow configurations during the course of flame propagation.
\end{abstract}

Key Words: LES, dynamic flame surface density, turbulent premixed flames, influence of the obstacles, Propane/air mixture 


\section{Introduction}

Large Eddy Simulation (LES) is gradually replacing Reynolds-averaging (RANS) method of solving the Navier-Stokes equations to compute the structure of turbulent flames. Several recent works confirm the high fidelity nature of LES in predicting key characteristics of complex reacting flows including those of practical combustors [1-5]. The main attraction of LES lies in its ability to fully resolve features of the flow above a certain cut-off length scale hence, making it possible to compute transient dynamics; this being a clear advantage over RANS methods. The penalty, however, lies in the additional computational cost and the need to model the unresolved contributions, hence the issue of sub-grid-scale (SGS) modelling. This is, particularly, an important issue in LES modelling of turbulent premixed combustion given that chemical reaction occurs at the molecular level and hence needs to be modelled at the sub-grid scale.

A range of approaches to model combustion at the SGS are being pursued at varying degrees and relevance to the spectrum of turbulent combustion. The flamelet approach [6] was used by many researchers in the past in various forms [7-9] and, although limited to thin reaction zones, remains applicable to a wide range of applications. Recent developments of this approach involve flame generated manifolds (FGM) tabulated in terms of mixture fraction, reaction progress variable as well as other parameters such as a measure of flow strain [10]. Such formulations enable the application of flamelet modelling in premixed, non-premixed, as well as partially premixed flames. Two variations of the laminar flamelet approach are the Flame Surface Density (FSD) where a transport equation for the FSD is solved [11] and the thickened flamelet model which has been applied successfully by Poinsot et al. [12]. Recently, Di Sarli and co workers [13, 14] demonstrated the importance of FSD based SGS model [1] to predict explosions in a vented chamber using LES. SGS modelling approaches 
that are seen as alternatives to flamelets include the filtered density functions (FDF) [15], the conditional moment closure [16] and the linear eddy model (LEM) [17]. Each of these approaches suffers from different limitations that are currently the subject of intense research. It is worth pointing that the combined LES/LEM is a truly multi-scale approach that is also receiving considerable attention.

In this paper the LES approach is used together with a recently developed dynamic flame surface density (DFSD) model [18-20] to compute turbulent premixed flames propagating in a laboratory scale combustion chamber containing a range of built-in solid obstructions. Earlier studies $[20,21]$ using the same DFSD model showed promising results in computing key characteristics of the propagating turbulent premixed flames but with only three selected configurations. In the present study, the main focus is to analyse the physics associated with flame-solids interactions and extend the calculations to a wide range of configurations to explore aspects such as the effects of location and number of the solid obstacles as well as area blockage ratio. The calculated results are validated against measurements taken from a novel experimental test facility [22, 23]. Eight different flow configurations are studied both experimentally and numerically. Results reported here also explore the effects of the resulting turbulence intensity on the structure of the reaction zone as well as the burning rate.

This paper is organized as follows: Section 2 briefly describes the experimental combustion chamber. Details of the newly developed SGS-DFSD model used in the LES calculations are outlined in Section 3. Numerical predictions for four groups of configurations are compared with available experimental data and reported in Section 4. Results are discussed highlighting the merits and drawbacks of the used model while discussing flame dynamics and behaviour in these groups of flow configurations. Finally general conclusions from the present investigation are summarised in Section 5. 


\section{Combustion Chamber and Test Cases}

The combustion chamber, shown schematically in Figure 1a is only briefly described here and more details can be found elsewhere $[22,23]$. It has internal dimensions of 50x50x250 $\mathrm{mm}$ giving an overall volume of 0.625 Litres. Up to three turbulent generating grids (also referred to as baffle plates or simply obstacles) may be placed in the chamber at $20 \mathrm{~mm}$, $50 \mathrm{~mm}$ and $80 \mathrm{~mm}$ from the base. Each baffle plate consists of five strips, $4 \mathrm{~mm}$ wide, evenly separated by six gaps, $5 \mathrm{~mm}$ wide, thus creating an overall blockage ratio of 0.4 . Downstream of the baffle plates, a further obstruction with a square cross section may be placed such that its bottom surface is maintained at $96 \mathrm{~mm}$ from the base plate. Two obstruction sizes are used, a small one with a cross section of $12 \times 12 \mathrm{~mm}$ and a large one with a $25 \times 25 \mathrm{~mm}$ cross section. The blockage ratios of these square obstructions are $25 \%$ and $50 \%$, respectively.

The fuel used throughout these experiments is Liquefied Petroleum Gas, LPG $\left(88 \% \mathrm{C}_{3} \mathrm{H}_{8}\right.$, $10 \% \mathrm{C}_{3} \mathrm{H}_{6}$ and $2 \% \mathrm{C}_{4} \mathrm{H}_{10}$ by vol.) at an equivalence ratio of $\phi=1.0$. The mixture is ignited from rest and ignition is achieved by focusing the infrared output from a Nd:YAG laser $3 \mathrm{~mm}$ above the base. Laser timing is controlled by the Q-switch of the Nd:YAG laser and this marks the start of each experiment or time zero. Pressure is recorded using two Keller type PR21-SR piezo-electric pressure transducers with a range of 0 - 1 bar and a total error $<0.5 \%$ located in the base plate and in the wall of the chamber just upstream of the exit plane. Eight configurations are rendered using this test chamber as shown in Figure 1b. These are clustered into four different "groups" as shown in Table 1 to test the following aspects:

- The effects of increasing the number of baffle plates starting with one baffle plate farthest from the ignition source (Group 1, Configurations: 5-2-1)

- The effects of increasing the number of baffle plates starting with one baffle plate closest to the ignition source (Group 2, Configurations: 7-4-1) 
- The effects of using the same number of baffles plates (two) positioned at different locations (Group 3, Configurations: 2-3-4)

- The effects of using the same number of baffles plates (one) positioned at different locations (Group 4, Configurations: 5-6-7)

It should be noted here that configuration 0 with no baffle plates is not included in any of the groups discussed here but it is useful as a baseline case for rest of the cases considered here.

\section{Modeling and Numerical Issues}

The governing equations and other numerical details associated with the LES model adopted in this paper are detailed elsewhere [18-20] and only a brief description is given here. A grid resolution of 90x90x336 (2.7 million cells) is adopted in the present calculations, as further refinement to 3.6 million cells shows no significant improvement in the results [19] for the present configuration. The filter width $\bar{\Delta}$ is calculated using a box filter, which is generally related to grid resolution by $2.0(\delta x \delta y \delta z)^{1 / 3}$ and fits in with the finite volume discretisation. The SGS combustion model used is described below in detailed, considering its importance and novelty.

In LES, modelling the filtered chemical reaction rate in turbulent premixed flames is very challenging due to its non-linear relation with chemical and thermodynamic states, and is often characterized by propagating thin reaction layers which are thinner than the smallest turbulent scales. In the present simulations, the SGS chemical reaction rate, $\overline{\dot{\omega}}_{c}$ is the source term in the Favre filtered reaction progress variable equation (see Eq. 1) and this is modelled using the laminar flamelet concept. The filtered conservation equation for the reaction progress variable may be written as: 


$$
\frac{\partial \bar{\rho} \tilde{c}}{\partial t}+\frac{\partial\left(\bar{\rho} \tilde{u_{j}} \tilde{c}\right)}{\partial x_{j}}+\frac{\partial\left(\bar{\rho}\left(\widetilde{u_{j} c}-\tilde{u_{j}} \tilde{c}\right)\right)}{\partial x_{j}}=\frac{\partial}{\partial x_{j}}\left[\frac{\bar{\mu}}{\operatorname{Sc}} \frac{\partial \tilde{c}}{\partial x_{j}}\right]+\overline{\dot{\omega}_{c}}
$$

In the above equation, $\rho$ is the density, $c$ is the reaction progress variable, $u_{j}$ is the velocity component in $x_{j}$ direction, $\mu$ is the dynamic viscosity, Sc is the Schmidt number and $\dot{\omega}_{c}$ is the rate of chemical reaction. An over-bar describes the application of the spatial filter, while the tilde denotes Favre filtered quantities. The reaction rate in Eq. 1 is modelled as:

$$
\overline{\omega_{c}}=\langle\rho u\rangle_{s} \bar{\Sigma}=\rho_{u} u_{L} \bar{\Sigma}
$$

where $\rho_{u}$ is the density of unburned mixture, $u_{L}$ is the laminar burning velocity, and $\bar{\Sigma}$ is the flame surface density (FSD). The filtered FSD term in Eq. $2(\bar{\Sigma}=\overline{|\nabla c|})$, can be split into two terms as resolved and unresolved:

$$
\bar{\Sigma}=\underbrace{\Pi(\bar{c}, \bar{\Delta})}_{\text {Resolved }}+\underbrace{\lambda(\bar{c}, \bar{\Delta}, \Pi(\bar{c}, \bar{\Delta}))}_{\text {Unresolved }}
$$

The resolved term in the above equation is evaluated as [24]:

$$
\Pi(\bar{c}, \bar{\Delta})=4 \sqrt{\frac{6}{\pi}} \frac{\tilde{c}(1-\tilde{c})}{\bar{\Delta}}
$$

The unresolved term in Eq. 3 is evaluated using the following expression:

$$
\lambda(\bar{c}, \bar{\Delta}, \Pi(\bar{c}, \bar{\Delta}))=\bar{\Sigma}-\Pi(\bar{c}, \bar{\Delta})=\overline{\nabla c \mid}-\Pi(\bar{c}, \bar{\Delta})
$$

The ratio of test filter to grid filter, i.e. $\hat{\bar{\Delta}} / \bar{\Delta}$ is defined as $\gamma$, such that the test filter $\hat{\bar{\Delta}}$ is greater than the grid filter $\bar{\Delta}$. In this study, test filter to grid filter ratio is considered as 2.0. Applying the test filter to FSD i.e. to equation 3 gives: 


$$
\widehat{\bar{\Sigma}}=\widehat{\mid \overline{\nabla c \mid}}=\underbrace{\Pi(\hat{\bar{c}}, \hat{\bar{\Delta}})}_{\text {Resolved@testfilter }}+\underbrace{[\widehat{|\nabla c|}-\Pi(\hat{\bar{c}}, \hat{\bar{\Delta}})]}_{\text {Unresolved@testfilter }}
$$

From the above equation, contribution of the unresolved-FSD ${ }^{2}$ at the test filter level can be written as:

$$
\Lambda=[\widehat{\mid \overline{\nabla c \mid}}-\Pi(\hat{\bar{c}}, \hat{\bar{\Delta}})]
$$

Assuming that the SGS contribution of unresolved-FSD as the difference of the SGS contributions, at test and grid filter and relating $\lambda$ and $\Lambda$ by using the Germano identity [25] gives:

$$
\begin{gathered}
\Lambda-\widehat{\lambda}=[\widehat{\mid \overrightarrow{\nabla c \mid}}-\Pi(\hat{\bar{c}}, \hat{\bar{\Delta}})]-[\widehat{\mid \overrightarrow{\nabla c \mid}}-\widehat{\Pi(\bar{c}, \bar{\Delta})}] \\
\Lambda-\widehat{\lambda}=[\widehat{\Pi(\bar{c}, \bar{\Delta}})-\Pi(\hat{\bar{c}}, \hat{\bar{\Delta}})]
\end{gathered}
$$

In the present simulations the SGS flame surface density contributions from the above equation can be added to the resolved-FSD with a model coefficient $C s$. Hence the total-FSD can be expressed as:

$$
\bar{\Sigma}=\Pi(\bar{c}, \bar{\Delta})+C s[\widehat{\Pi(\bar{c}, \bar{\Delta}})-\Pi(\hat{\bar{c}}, \hat{\bar{\Delta}})]
$$

The model coefficient $C s$ in above equation is dynamically obtained by identifying sub-grid flame surface as a fractal surface [2].

$$
C s=\frac{1}{1-\gamma^{2-D}}\left[\left(\frac{\bar{\Delta}}{\delta_{c}}\right)^{D-2}-1\right]
$$

\footnotetext{
${ }^{2}$ Unresolved-FSD: FSD due to unresolved progress variable in the solution field.
} 
where $\delta_{c}$ is the lower cut-off scale, taken equal to three times of the laminar flame thickness [2] and $D$ is the fractal dimension, calculated dynamically [2] as:

$$
D=2.0+\frac{\log (\langle\widehat{\Pi(\bar{c}, \bar{\Delta}})\rangle /\langle\Pi(\hat{\bar{c}, \hat{\bar{\Delta}}})\rangle)}{\log (\hat{\bar{\Delta}} / \bar{\Delta})}
$$

In the above equation, angular brackets \langle\rangle denotes conditional averaging within the flame bounded by $\tilde{c}=0$ to $\tilde{c}=1$. The numerical model described above is implemented in an inhouse, compressible version of the LES code called PUFFIN [26].

The simulations are performed using an initially stagnant propane/air mixture of equivalence ratio 1.0. The code solves fully compressible, strongly coupled, Favre-filtered flow equations discretized using a finite volume method. The discretization is based on control volume formulation on a staggered non-uniform Cartesian grid. A second order central difference approximation is used for diffusion, advection and pressure gradient terms in the momentum equations and for gradient in the pressure correction equation. Conservation equations for scalars use second order central difference scheme for diffusion terms. Third order upwind scheme of Leonard, QUICK [27] and SHARP [28] are used for advection terms of the scalar equations to avoid problems associated with oscillations in the solution. The QUICK scheme is also sometimes used for the momentum equations in areas of the domain where the grid is expanded and accurate calculation of the flow is less important. The equations are advanced in time using the fractional step method. Crank-Nicolson scheme is used for the time integration of momentum and scalar equations. A number of iterations are required at every time step due to strong coupling of solved equations.

The computational domain is shown in Figure 1c. It should be noted here that LES simulations presented in this paper were carried out using the $12 \times 12 \mathrm{~mm}$ square obstruction. 
The combustion chamber has dimensions of 50x50x250 mm where the flame propagates over the baffles and solid obstacle. Solid boundary conditions are applied at the bottom, vertical walls, for baffles and obstacle by setting the normal and tangential velocity components to zero, which ideally represents impermeable and no-slip conditions. The walls and obstacles are considered to be isothermal and the same temperature is maintained thorough out the simulations. The wall shear is calculated by the $1 / 7^{\text {th }}$ power-law wall function of Werner and Wengle [29] taking the form of $\tau_{w}=W(\tilde{u}, y)$, where $\tau_{w}$ is the wall shear stress, $W$ is a functional dependence, $y$ is the distance of the grid point form the wall and $\tilde{u}$ is the tangential velocity at $y$. Outflow boundary condition is used at the open end of the combustion chamber. To ensure that the outflow boundary condition at the open end of the domain is accurate and allows the pressure waves generated within the chamber to leave the computational domain without reflection, the numerical domain is adequately extended to $325 \mathrm{~mm}$ in $x, y$ and $250 \mathrm{~mm}$ in $z$ direction with a large grid expansion ratio of approximately 1.25 . A nonreflecting boundary condition [26] analogous to commonly used convective boundary condition, in incompressible LES is used to prevent reflection of pressure waves at this boundary. The initial conditions are quiescent with zero velocity and reaction progress variable. Ignition is modelled by setting the reaction progress variable to 0.5 within a radius of $4 \mathrm{~mm}[30]$ at the bottom centre of the chamber, in order to achieve the initial quasi laminar phase corresponding to experiments.

The governing equations, discretized by the finite volume method, are solved using a BiConjugate Gradient solver with an MSI pre-conditioner for the momentum, scalar and pressure correction equations. The time step is limited to ensure the CFL number remains less than 0.5 with the extra condition that the upper limit for $\delta t$ is $0.3 \mathrm{~ms}$. The solution for each time step requires around 8 iterations to converge, with residuals for the momentum 
equations less than $2.5 \mathrm{e}-5$ and scalar equations less than 2.0e-3. The mass conservation error is less than $5.0 \mathrm{e}-8$.

\section{Results and Discussions}

Results are presented in this section for the base flow configuration followed by those for the four groups of cases listed in Table 1. Calculations are compared with available averaged measurements which include pressure-time traces, mean and rms fluctuations of velocity, as well as high-speed video images of flame emission (2000 frames per second). It should be noted that the high-speed video images are not space-resolved and mark the leading edge of the propagating flame front. These are compared with calculated reaction rates to validate the location of the flame leading edge and the global degree of contortion in the flame. Video images from experiments are only available up to $25 \mathrm{~cm}$ of the test facility and no information is available after this. It is worth mentioning at this stage that all experimental measurements are highly repeatable. For example, Figure 2 a shows five individual pressure-time histories of experimental measurements for configuration 1 together with averaged curve in black solid line. The variation and standard deviation of overpressure are calculated as $11.46 \mathrm{mbar}(<$ $10 \%$ ) and $3.38 \mathrm{mbar}$ respectively. The variation of peak overpressure occurrence (time) is $0.02 \mathrm{~ms}$. The variation of peak overpressure in other configurations is $<5 \%$. Figure $2 \mathrm{~b}$ shows axial velocity data for configuration 3 from over 50 individual experiments together with mean velocity obtained using an ensemble averaging technique with a time interval or bin size of a quarter of a millisecond [31]. It should be noted here that the measurements were carried out at the middle of the chamber ( $x$-axis) and half-way up the side of the square obstacle i.e. $102 \mathrm{~mm}$ from the base ( $z$-axis) and $16 \mathrm{~mm}$ from the central axis ( $y$-axis) using the Laser Doppler Velocimetry (LDV) technique. The mean profile obtained is further processed 
using a least squares fit polynomial to alleviate the errors imbedded in the flow data arising from the ensemble mean.

\subsection{Base Flow Configuration}

The base flow configuration, noted here as configuration 0 , has no internal baffle plates, but include a built-in solid square obstacle $(12 \times 12 \mathrm{~mm}$ cross section) running across the chamber such that its base is $96 \mathrm{~mm}$ from the ignition end. Time series of the overpressure and the flame position from LES are plotted against experimental measurements in Figure 3. Since, solid baffle plates are not present in this configuration; the flame travels relatively slowly as seen from both measurements and calculations. The overpressure trend from LES is in excellent agreement with experimental measurements for the duration of flame travelling from ignition to the exit of the chamber. The time at which pressure rises is at about $11.5 \mathrm{~ms}$ and peak overpressure occurs at about $13.5 \mathrm{~ms}$. The LES predicts a peak overpressure of $36.6 \mathrm{mbar}$ at $13.5 \mathrm{~ms}$ compared to a measured value of $34 \mathrm{mbar}$ at $13.5 \mathrm{~ms}$ producing an overprediction of about 7.6\%. It is evident from Figure 3 that the pressure reflections once the main flame left the chamber are in reasonable agreement with the experiments. It is observed that the trapped unburned mixtures up and down stream of the solid obstacle are consumed once the main flame leaves the chamber leading to subsequent pressure oscillations. The flame position shown in Figure 3 confirms this observation with a good match of up to the peak overpressure and thereafter with a slight, but considerable deviation.

Figure 4 shows a sequence of images for the calculated reaction rate contours compared to the measured high-speed video images at various times after ignition. It is evident from these images, that the LES technique is capable of reproducing turbulent flame structure and propagation rates with high level of accuracy. For instance, the semi-circular shape of the flame is gradually flattened as it approaches the obstacle as seen in the images at $12 \mathrm{~ms}$. 
Similarly, at $13.5 \mathrm{~ms}$ (peak overpressure incidence) both LES and measurements demonstrate the entrainment of unburnt mixture on the upstream edge of the obstacle as it is engulfed by the flame. This trapped mixture is seen to burn before $14.5 \mathrm{~ms}$. However, there is some unburnt mixture trapped in the formed recirculation zone downstream the solid obstacle and this burns after the leading edge of the flame has exited the chamber. It is also noted from Figure $4 \mathrm{a}$ that the predicted turbulent flame thickness is about 1 to 3 computational grid widths and the development of flame stretch is clearly noticed once the flame starts interacting with the square obstacle.

Figure 5 shows the measured mean and rms values for the axial and radial flow velocity components plotted against corresponding LES results at the same location defined in section 4. Experimental axial (W) and radial (V) velocity measurements shown in Figure 5 are the ensemble averaged velocity data from over 50 repeatable individual experiments with a timebin of $0.25 \mathrm{~ms}$ [31]. Accordingly, the rms fluctuations of $\mathrm{W}$ and $\mathrm{V}$ are estimated from the variance between a polynomial fitted to the ensemble mean velocity data.

In case of LES, the data are available from only one simulation where the flame is totally unsteady, so ensemble averaged velocity information is not available. The only alternative choice is to take a suitable calculation time span (time-bin) over which mean and rms fluctuations can then be obtained. From the LES calculations, there exists a large number of data points $(\sim 100$ to 500$)$ for every one millisecond of the flow due to the limitation of Courant-Friedrichs-Lewy (CFL) condition. Therefore, a time-bin size of $0.25 \mathrm{~ms}$ is chosen to extract average and its variance at the same location in the chamber.

It is evident from Figure 5a that the maximum axial velocity has reached $32 \mathrm{~m} / \mathrm{s}$ at approximately $14.5 \mathrm{~ms}$ as obtained from both experiments and LES. Mean and rms velocity profiles of the LES are in reasonable agreement up to approximately $15 \mathrm{~ms}$ after ignition but 
deviate slightly thereafter. Notwithstanding the uncertainty in the measurements, the rms fluctuations in Figure 5a provide good estimates of the turbulence levels at various stages of flame propagation. It can be seen that, the rate of increase in turbulence and its decay is fairly well predicted at peak pressure incidence i.e. $13.5 \mathrm{~ms}$ and during the blow-down phase.

Noting that this flow is a highly dynamic and transient combustion situation the peak values of the mean and rms of radial velocities shown in Figure $5 \mathrm{~b}$ are in adequate agreement between LES and measurements. The computed mean velocity trend is in good agreement with experimental trend. However, the computed rms fluctuations show some disagreement with measurements. This is expected considering that neither measurements nor LES calculations of rms are presenting true values of local turbulence. The experimental rms includes shot-to-shot variations associated with the repeatability of the measurements. It is worth noting here that given the transient nature of the flame, measuring true turbulence is not a trivial task as experienced by other researchers too in the past [32] and requires a technique with data collection ability in the range of tens of $\mathrm{kHz}$.

\subsection{Overview of Groups 1 to 4}

In order to facilitate the discussion in the remainder of this paper, overall trends obtained from the various groups of configurations are first presented. Figure 6 show plots of the peak overpressure and the time to reach the peak (referred to hereon as ("time-to-peak") for Groups 1 to 4 . It is evident from Figure 6a that, as expected, the overpressure generated is higher and the time-to-peak is shorter in the case of configuration 1 since it has 3 baffle plates. It is very interesting to note that the time-to-peak in this group of experiments maintains a linear relation within the group while overpressure is non-linear as seen in Figure 6a. The magnitude of the overpressure is approximately increased by $50 \%$ and $75 \%$ due to the addition of one and two baffles in configurations $2 \& 1$ respectively, when compared to 
the overpressure of configuration 5, which has only one baffle plate. However, the time-topeak decreased by $11.5 \%$ and $23 \%$ in configurations $2 \& 1$ respectively. The non-linear relation of the peak overpressure in this group is postulated to be due to the increase in baffle plates and their respective positions in the configurations.

Figure $6 \mathrm{~b}$ presents overpressures and time-to-peak for Group 2. It is evident that both overpressure and time-to-peak are non-linear in this group. The overpressure has increased by $71 \%$ and $206 \%$ in configurations 4 and 1 respectively compared to the measured overpressure in configuration 7 . The time-to-peak has decreased by $11 \%$ and $6.4 \%$ in configurations $4 \& 1$, respectively, as compared to configuration 7 . The measured time-to-peak in configuration 4 is about $9.8 \mathrm{~ms}$ and found to be the fastest compared to any other configuration studied in this investigation. By comparing configurations $4 \& 1$ in Figure $6 b$, the increase in time-to-peak when relatively moving from configuration 4 to configuration 1 is clearly noticeable. It is evident that, the number of baffles in these configurations may increase the magnitude of the overpressure as noticed in Groups $1 \& 2$. Also, it is clear that the time-to-peak trend in Group 2 is different compared to Group 1 and highlights that the time-to-peak is mainly dependent on the relative position of the baffles to the ignition source in any configuration.

Figure $6 \mathrm{c}$ presents overpressures and time-to-peak in Group 3. It is very interesting to note that having two baffles at different positions in this group has produced different pressures and time-to-peaks. The recorded overpressure is found to be higher in configuration 2 and lower in configuration 4. Similarly the time-to peak is higher in configuration 2 and lower in configuration 4. Figure 6d presents overpressures and time-to-peak in Group 4. Experimental measurements for configurations $6 \& 7$ are not available to compare. However, indicative overpressure and incident time from the work of Hall et al. [20] using a rich mixture $(\phi=1.1$ 
) in the same test facility having one baffle and square obstacle at the same locations are used here. It should be noted that these measurements used here just to give an approximate indication.

Remarkably configuration 5 having one baffle plate at S3, away from ignition end, has resulted in higher overpressure of $71 \mathrm{mbar}$ at $13.3 \mathrm{~ms}$. Configuration 7 , which has one baffle plate at S1 near to the ignition bottom, has produced the lowest over pressure of 58mbar at $12.5 \mathrm{~ms}$. Configuration 6 , with one baffle plate at $\mathrm{S} 2$, has produced an overpressure of $65 \mathrm{mbar}$ at $12.1 \mathrm{~ms}$, which lies in between the other two configurations. The overall overpressure trends obtained in Groups 3 and 4 are found to be very similar. A slight difference in the measured and LES time-to-peak trends of Groups 3 and 4 are noticeable from Figures $6 \mathrm{c}$ and $6 \mathrm{~d}$ respectively. This is mainly thought to be due to the additional baffle plate and its respective position in Group 3 configuration. However, Groups 3 and 4 evidently confirm that the farther the location of the baffles from the ignition bottom, the higher the peak overpressure. Also, closer the baffles to the ignition bottom, the lesser the magnitude of peak overpressure. More details will be discussed in the next sections for individual groups of test cases.

\subsection{Results for Group 1 Test Cases}

Group 1 consists of configurations 5-2-1 with progressively increasing the number of baffle plates from one to three starting with the position farthest from ignition source as detailed in Table 1. The time histories of overpressure and flame position from LES and experiments are shown in Figures $7 \mathrm{a} \& 7 \mathrm{~b}$ respectively. It is evident from Figure $7 \mathrm{a}$ that the overpressure trend is in good agreement albeit slight under-predictions. Figure 7a also highlights the impact of the number of baffles and their position with respect to distance from the ignition 
source. In configuration 5, the time taken to reach the first baffle plate is long compared to configurations 2 and 1 and the turbulence generated in the latter two configurations is expected to be higher due to progressively higher blockage ratio leading to higher overpressure as evident from the results. Similarly, the flame position shown in Figure $7 \mathrm{~b}$ is also well predicted and the trends are as expected, that the flame propagates faster is configuration 1 as compared to configuration 5.

Computed flame speed and acceleration for configurations 5, 2 and 1 shown in Figures 8a \& $8 \mathrm{~b}$ are in good agreement with those derived from the measured flame images, except when the flame is located downstream of the square obstacle in the blow-down region. This may be partly due to the limited resolution of high-speed images. Within the blow-down region, the flow condition is highly turbulent and the flame propagates at speeds of about $80-100 \mathrm{~m} / \mathrm{s}$. At an imaging rate of 2000fps, the flame front could have moved about 8-10 $\mathrm{mm}$ between subsequent frames. It is worth noting here that high-speed imaging of laser induced fluorescence from reactive species such as $\mathrm{OH}(\mathrm{LIF-OH})$ would be extremely useful since such measurements could be made at a rate of $5 \mathrm{kHz}$ or higher and would be spatially resolved as well. Such capabilities are now available and LIF-OH imaging is planned for the configurations studied here.

Figure 9 presents the reaction rate contours computed at 6.0, 8.0, 10.0, 11.5 and $12.0 \mathrm{~ms}$ after ignitions for the three flames in Group 1. These plots, clearly, display the relative change in the flame structure with respect to the addition of baffle plates. For instance, the plots at 8.0 ms for configurations 2 and 5 (Figures 9a \& 9b) illustrates the semi-circular flame structure, which is generally expected in chambers having length to diameter ratio greater than 3 . At subsequent times, the finger-like structures of the flame protruding from the channels of the baffle plates are visible in all configurations. Figure $9 \mathrm{~b}$ at 11.5 and $12.0 \mathrm{~ms}$ shows a clear 
picture of entrapment of unburned fuel/air mixture around the solid square obstacle within the recirculation zone. Figure 9c shows that the pockets of trapped unburned mixture in the case of configuration 1 occurring at 10.0 and $11.5 \mathrm{~ms}$ are clearly noticeable. These extended simulations have not been performed for all configurations due to computational resource limitations. Similarly, Figure $9 \mathrm{c}$ at 11.5 and $12.0 \mathrm{~ms}$ shows the consumption of trapped mixture, once the main flame left the chamber. Figure 9 also quantifies the influence of baffles on overpressure and turbulence generation through the flame. This mixture is consumed once the main flame has left the chamber causing pressure oscillations as observed experimentally. The LES calculations found to capture these oscillations as shown in configuration 0 (Figure 3).

The current work shows a clear improvement over earlier attempts made to calculate the detailed flame structure [18] and this is believed to be largely due to the addition of unresolved FSD contribution at SGS level. The contribution of the unresolved FSD is expected to increase with turbulence levels as evident from the plots of Figure 10a which shows the unresolved FSD plotted against turbulence fluctuation normalised with laminar burning velocity $\left(u^{\prime} / u_{L}\right)$. The velocity fluctuations are calculated as mentioned above in Section 4.1. It can be noted that the trend is consistent for all three configurations where the unresolved FSD sharply increases with higher values of $u^{\prime} / u_{L}$. The ratio of the unresolved to the resolved FSD is found to be proportional to the turbulence intensity at any given instance. It is noted that the contribution of the unresolved FSD ranges from 15 to $50 \%$ of the resolved FSD [33] and hence must be included. It is interesting to note that for values of $u^{\prime} / u_{L}>20$ all configurations show a steeper rate of increase in the unresolved FSD; the highest being for configuration 5. This might be one of the reasons for the under-prediction of overpressure 
and the slight shift of peak overpressure observed earlier. It should be noted that the estimates of turbulence and FSD reported here are obtained at the leading edge of the flame.

Figure $10 \mathrm{~b}$ shows the combustion regimes for configurations 5, 2 and 1 on a standard regime diagram [34] for turbulent premixed flames. While results are shown for the leading edge of the flame in each configuration, they illustrate the transition into different regimes as the reaction zone propagates through different turbulence levels. Following ignition, during the early stages of flame propagation, the flame is found to be within the wrinkled and corrugated flamelet regimes for all three configurations. However, for most of its lifetime, the flame lies in the thin reaction zone regime, where flamelet models are applicable. The findings of Di Sarli et al. [10] for combustion in a rectangular chamber with multiple obstacles supports the current observations. As turbulence level increases, it is likely that the flame enters into the broken reaction zone and hence the laminar flamelet modelling approach would no longer be valid. Such occurrences, while possible, are not likely to be frequent in the present flow configurations.

To elaborate this further, the range of turbulence levels, corresponding combustion regimes and the Karlovitz numbers and corresponding times spent under each regime for all three configurations of this group are presented in Table 2. It is interesting to note that in all configurations the flame progresses from wrinkled flamelet regime to the thin reaction zone regime through a corrugated flamelet regime (see Figure 10b). The time spent within the wrinkled flamelet regime by the flame in each configuration generally corresponds to how long the flame maintains its laminar structure after ignition (see Figure 10b). From Table 2, it is evident that the time spent by the flame within the corrugated flamelet regime in configuration 5 is longer than that of configuration 1 . This observation confirms that the 
flame is passing through various regimes of turbulent premixed combustion as it interacts with different turbulence levels. However, it can be confirmed that the flame in these configurations did not enter into the broken zone regime.

Figure 10c shows the time derivatives of the resolved and unresolved flame surface areas plotted against averaged Karlovitz number for the entire lifetime of flame propagation in configuration 1. The Karlovitz number is defined here based on the LES filter width [35]. Computed Karlovitz numbers within the reaction zone bounded by reaction progress variable of 0 to 1 are averaged in the middle plane across the chamber. It is worth noting that, as the flame propagates, the turbulence level progressively increases. However, the rate at which the flame surface area grows is not uniform with Karlovitz number. From Figure 10c, it is evident that absolute maximum of rate of resolved flame surface $(d A / d t)$ occurs at a Karlovitz number of $\mathrm{Ka}=61$ and corresponding to $10.1 \mathrm{~ms}$ of flame evolution (time is not shown in plots). However, absolute maximum of rate of unresolved flame surface $(d A / d t)$ occurs at a Karlovitz number of $\mathrm{Ka}=72$ and corresponding to $10.2 \mathrm{~ms}$. This behaviour may be due to two reasons. Firstly, the Karlovitz number presented in Figure 10c is averaged as described above. Secondly, after the flame leading edge leaves the chamber, the development of the combustion is very much restricted to the trapped unburnt gases. Therefore, Figure 10c would be more meaningful within the Karlovitz number below 100 .

\subsection{Group 2 Test Cases}

Configurations 7-4-1 forming Group 2 have similar number of baffle plates as Group 1 except that the arrangement starts close to the ignition end as detailed in Table 1 . Since the details of configurations $7 \& 4$ are being discussed in next sections, they are not discussed here to avoid repetition. Only one additional comment is made here. 
In general, overpressure increases with the number of blockages (see Figure 6b). However, the rise of peak overpressure from configurations 4 to 1 and configuration 2 to 1 is very noticeable (see Table 1 and Figures $6 a \& 6$ b). This finding is certainly helpful in positioning the blockages (any equipment or workstations) in industrial environments or commercial buildings, in order to reduce the risk of overpressure generated in an accidental explosion. Recent explosion investigation reports of Buncefield oil depot, Hemel Hempstead, UK [36] and Hayes Lemmerz International (HLI), Huntington, Indiana, USA [37], clearly identified the influence of blockages on overpressure and highlighted the damage caused by the generated overpressure. The Buncefield investigators identified that few 100 mbar of generated overpressure in this explosion incident has caused severe damages as shown in their report [36]. The HLI investigators recommended increasing the gap between the dust collectors and the foundry building to 50 feet and felt that the previous arrangement had increased the risk to personnel during explosion. Similar considerations could be given in the design of offshore drilling platforms.

\subsection{Group 3 Test Cases}

Group 3 has three configurations 2-3-4 with two baffle plates at different positions and the solid square obstacle at the fixed position. Figure 11a shows good agreement between predictions and measurements of the rate of pressure rise and trend, including the first hump. However, in configuration 4 , the computed rate pressure rise is slower than measurements, which indicates a faster decay of turbulence being predicted between the second baffle and the square obstacle. Figure $11 \mathrm{~b}$ shows the calculated and measured flame position in this group. In configuration 3, the computed flame position is in excellent agreement with measurements. The agreement in configurations $2 \& 4$ is good. However, faster flame 
propagation is predicted during the blow-down phase, where highest level of turbulence is present.

Figures $12 \mathrm{a} \& 12 \mathrm{~b}$ shows comparisons between the computed and measured flame speed and acceleration in this group. The highest flame speed and acceleration are recorded for configuration 2. It should be noted that, configuration 4 has the lowest overpressure, flame speed and acceleration. It is believed that this is largely due to the longer separation between the second baffle plate and the obstacle. Such large separation allows the flame to relaminarise and hence lower its speed of propagation.

Figure 13 shows the reaction rate contours and corresponding video images at various instances in this group. The flame-obstacles interactions in this group can be analysed by combining the results presented in Figures 12 and 13. For instance at $6 \mathrm{~ms}$, the flame is seen to be jetting out of the first baffle in configurations $3 \& 4$. However at $6 \mathrm{~ms}$, the flame is seen to be relatively smooth in configuration 2 . This observation is supported by the axial rms velocity presented in Figure 14 of $2 \mathrm{~m} / \mathrm{s}$ in configurations $3 \& 4$ against $0.2 \mathrm{~m} / \mathrm{s}$ in configuration 2 at $6 \mathrm{~ms}$.

Similarly, the flame in configurations $2 \& 3$ can be seen to be interacting with the baffle plate at S3 leading to a different flame structure at $10 \mathrm{~ms}$. The axial $\mathrm{rms}$ velocities are found to be 4 and $5 \mathrm{~m} / \mathrm{s}$ respectively in the above configurations. However at $10 \mathrm{~ms}$, the flame in configuration 4 is found to be more turbulent with axial rms velocity of $8 \mathrm{~m} / \mathrm{s}$. Figures 13 and 14 also illustrate quicker flame exit in configuration 4 than in configuration 2 . Though, the flame in configuration 2 is at lower speed at the beginning, it became highly turbulent due to jetting and contortion through repeated baffles. The rms of the axial velocity component in this configuration is found to be progressively increasing and reaches a maximum of $9 \mathrm{~m} / \mathrm{s}$ at $11.5 \mathrm{~ms}$. 
In configuration 3, the flame is found to be distorted as it reaches the first baffle. However, re-laminarisation of the flame between $\mathrm{S} 1$ and $\mathrm{S} 3$ slows the speed of propagation between $\mathrm{S} 1$ and S3 such that the flame front reaches the obstacle at a later stage compared to configuration 4 . This is also evident from the computed rms fluctuations at $10 \mathrm{~ms}$ as 5 and 8 $\mathrm{m} / \mathrm{s}$ and at $11.5 \mathrm{~ms}$ as 5 and $7 \mathrm{~m} / \mathrm{s}$ respectively in configurations 3 and 4.

\subsection{Group 4 Test Cases}

Group 4 consists of configurations 5-6-7 with only one baffle positioned at different locations from the ignition end with the square obstacle. Figures $15 \mathrm{a} \& 15 \mathrm{~b}$ presents the time histories of overpressure and flame position respectively in this group. Evidently, Figure 15a illustrates the agreement between computed and measured overpressure in configuration 5. From numerical predictions, it is interesting to note that the configuration 7 has recorded the lowest overpressure compared to configuration 5 in this group. This is believed to be due to the position of baffle plate in the chamber from the ignition end. As the distance between the baffle plate and square obstacle in configuration 7 is longer compared to configuration 5 , relaminarisation of the flow might have occurred.

In order to examine the flame-obstacle interactions, the computed reaction rate at 5 instances i.e. $6,8,10,12$ and $13 \mathrm{~ms}$ are presented in Figure 16. These time instants are generally matched with the flame evolution, interactions with baffle and square obstacle, formation of recirculation zone and flame blow-down from the chamber.

At 6 and $8 \mathrm{~ms}$, configurations $5 \& 6$ are found to have very similar flame structure and shape with approximately the same flame thickness and reaction rate. Since configuration 7 has a baffle plate at $\mathrm{S} 1$, the flame is found to be jetted through the baffle, which eventually changes the flame shape. The flame position at $8 \mathrm{~ms}$ in configuration 7 is almost equal to the other two 
configurations in this group (see Figure 15b). However at 10ms, the flame in configuration 7 is found to have a higher surface area. At $10 \mathrm{~ms}$, the flame in configuration 6 is found to evolve through baffle slits and starts to form individual flame kernels. However, the flame in configuration 5 at $10 \mathrm{~ms}$ is still smooth and is found to have a finger shaped structure, propagating in the axial direction at a rate proportional to the gas expansion ratio (defined as the density ratio of the fresh and burned fuel/air mixture).

Reaction rate contours at $12 \mathrm{~ms}$ in Figure 16 are very interesting and delineate information about the flame entrapment around the square obstacle. Comparison of the reaction rate in configurations $6 \& 7$ at $12 \mathrm{~ms}$ confirms that the flame in configuration 7 has more flame surface area with smooth outer and wrinkled inner flame structure. The inner flame structure is responsible for trapping of unburned mixture. However, the flame in configuration 6 has smooth inner structure, which engulfs a lesser amount of the mixture. Also, some flame islands can be observed in configuration 6, which are believed to be responsible for slightly higher overpressure at any given time compared to configuration 7 .

Comparing reaction rate contours at $13 \mathrm{~ms}$ in configuration 5 , with the contours at $12 \mathrm{~ms}$ in configurations $6 \& 7$, enhances our understanding of how, individual flow configuration traps the unburned mixture. Figure 16 also reveals that the mixture trapped in configuration 5 is much less compared to configurations $6 \& 7$. This is believed to be due to the gap between the baffle and the square obstacle, which has a greater influence on the turbulence intensity.

\section{Conclusions}

Measurements and LES simulations have been carried out for propagating turbulent premixed flames in eight different flow configurations employing a newly developed SGS-DFSD model. A stagnant, stoichiometric propane/air mixture was used in the current investigation. All flow configurations were clustered into four groups based on the number and position of 
baffles, in order to understand the underlying complex mechanism of flame-flow-obstacle interactions. Main conclusions from the current study can be categorized as follows:

\subsection{Improvements in the Predictions due to the SGS-DFSD model:}

In general, the LES predictions for all flow configurations are in good agreement with experimental measurements of the flame structure, position and speed as well as the reaction zone development. The time histories of the generated overpressure are in excellent agreement with measurements in all test groups. The overpressure, which generally represents the generated energy in any individual configuration, was identified to be proportional to the number of solid obstructions and their relative location to the ignition end.

In the selected flow configurations, considered here, majority of the combustion is within the thin reaction zone regime of turbulent premixed combustion. A progressive increase of the unresolved FSD contribution with increased turbulence level is identified and this highlights the importance of the unresolved FSD in the employed model in achieving accurate predictions.

\subsection{Improved Understanding of the Flame-Flow-Obstacle Interactions:}

The blockage ratio, relative position and number of the solid obstructions, and their relative position to the ignition source found to have a significant impact on the magnitude of overpressure and the spatial flame structure. Extensive flame-flow interactions found to occur as the flame propagates past the baffle plates and solid obstacle. This has resulted in a progressive increase in acceleration. The formation of few disconnected flame islands are attributed to the presence of the solid obstructions and formation of recirculation zones. That is where the trapped unburnt mixture within these islands is burned at a faster rate. Moreover, 
the trapped unburned mixtures, up- and down-stream of the solid square obstacle are consumed once the main flame exit the chamber, causing pressure oscillations. Although, high turbulent burning velocities of about 12 to $14 \mathrm{~m} / \mathrm{s}$ near the open end of the chamber were noticed in some configurations, no evidence of flame quenching due to elongation, stretching or distortion in the present study.

Large separation between solid baffles in all flow configurations, studied here, found to allow for sufficient residence time for turbulence decay. This has resulted in a lower overpressure and less distortion in the reaction zone, leading to a much smoother flame front. Extending the distance between solid baffles furthermore, will allow turbulence to decay to a much greater extent, even with the same blockage capacity. This new finding highlights the transient nature of the interaction between propagating flames and local flow field. Finally, it is found that location of the solid obstacles with respect to the ignition source has a direct impact on the level of the generated pressure and the reaction zone structure.

\section{References}

[1].F. Charlette, C. Meneveau, D. Veynante, Combust. Flame 131 (1-2) (2002) 181-197.

[2].R. Knikker, D. Veynante, C. Meneveau, Phys. Fluids 16 (11) (2004) L91-L94.

[3].C. Fureby, H. Pitsch, Lipatnikov, E. Hawkes, Proc. Combust. Inst. 30 (1) (2005) 593-601.

[4].A.R. Masri, S.S. Ibrahim, B.J. Cadwallader, Exp. Therm. Fluid Sci. J. 30 (7) (2006) 687702.

[5].H. Pitsch, Ann. Rev. Fluid Mech. 38 (2006) 453-482.

[6].K.N.C. Bray, M. Chalmpion, P.A. Libby, Turbulent Reacting Flows, Springer Publications, New York, 1989.

[7].E. Knudsen, S. H. Kim, and H. Pitsch, Phys. Fluids 22 (11) (2010) 115109.

[8].J. A. Van Oijen, L. P. H. de Goey, Combust. Theory and Modelling, 6 (3) (2002) 463 478.

[9].A. W. Vreman, J.A. Van Oijen, L.P.H. de Goey, R. Bastiaans, Flow, Turbulence and Combust. 82 (4) (2009) 511-535.

[10]. A.W. Vreman, B.A. Albrecht, J.A. van Oijen, L.P.H. de Goey, R.J.M. Bastiaans, Combust. Flame, 153 (2008) 394-416.

[11]. E.R.Hawkes, R.S. Cant, Combust. Flame, 126 (2001) 1617-1629.

[12]. O. Colin, F. Ducros, D. Veynante, T. Poinsot, Phys. Fluids, 12 (2000) 1843-1863. 
[13]. V. Di Sarli, A. Di Benedetto,G. Russo, S. Jarvis, E.J. Long,G.K.Hargrave, Flow Turbul. Combust. 83 (2009) 227-250.

[14]. V. Di Sarli, A. Di Benedettoa, G. Russo, J. Hazard. Mater. 169 (2009) 435-442.

[15]. S.I. Möller, E. Lundgren, C. Furbey, Proc. Combust. Inst., 26 (1996) 241-248.

[16]. R.W. Bilger, Phys Fluids, A 5 (2), (1993) 436-444.

[17]. P.A. McMurthy, S. Menon, A.R. Kerstein, Proc. Combust. Inst., 24 (1992) 271-278.

[18]. S.R. Gubba, S.S. Ibrahim, W. Malalasekera, A.R. Masri, Combust. Sci. Technol. 180 (10) (2008) 1936-1955.

[19]. S.R. Gubba, S.S. Ibrahim, W. Malalasekera, A.R. Masri, Combust. Theory and Modelling, 13 (2009) 513-540.

[20]. S.R. Gubba, S.S. Ibrahim, W. Malalasekera, in: $6^{\text {th }}$ Mediterranean Combustion Symposium, Ajaccio, Corsica, France, 2009.

[21]. S.S. Ibrahim, S.R. Gubba, AR Masri, W Malalasekera, J. Loss Prevent. Proc. Ind. 22 (2009) 258-267.

[22]. J.E. Kent, A.R. Masri, S.H. Starner, in: 5th Asia-Pacific Conference on Combustion, The University of Adelaide, Adelaide, Australia, 2005.

[23]. R. Hall, A.R. Masri, P. Yaroshchyk, S.S. Ibrahim, Combust. Flame 156 (2009) 439446.

[24]. R. Knikker, D. Veynante, C. Meneveau, Proc. Combust. Inst. 29 (2002) 2105-2111.

[25]. M. Germano, U. Piomelli, P. Moin, W.H. Cabot, Phys. Fluids A 3 (7) (1991) 17601765.

[26]. M.P. Kirkpatrick, S.W. Armfield, A.R. Masri, S.S. Ibrahim, Flow Turbulence Combust. 70 (1) (2003) 1-19.

[27]. B.P. Leonard, in: Comp. Methods in Applied Mech. Eng. (1979).

[28]. B.P. Leonard, Sharp simulation of discontinuities in highly convective steady flow. NASA technical memorandum, 100240, (1987).

[29]. H. Werner, H.Wengle, in: 8th Symposium on Turb. Shear Flows, Munich, Germany, (1991).

[30]. D. Bradley, F.K.K. Lung, Combust. Flame 69 (1987) 71-93.

[31]. R. Hall, Influence of obstacle location and frequency on the propagation of premixed, M.E. thesis, The University of Sydney, Sydney (2008).

[32]. R. P. Lindstedt and V. Sakthitharan, Combust. Flame 114 (1998) 469-483.

[33]. S.R. Gubba, Development of a dynamic LES model for premixed turbulent flames" PhD thesis, Loughborough University, UK (2009).

[34]. N. Peters, Turbulent Combustion, Cambridge University Press, 2000.

[35]. H. Pitsch, D. De Lageneste, Proc. Combust. Inst., 29 (2002) 2001-2008.

[36]. The Buncefield Incident 11 December 2005 The final report of the Major Incident Investigation Board, Published on 11 Dec 2008, ISBN-9780717662708, (http://www.buncefieldinvestigation.gov.uk/reports/index.htm).

[37]. Hayes Lemmerz International Investigation Report, Aluminum Dust Explosion, Published on September 2005, REPORT NO. 2004-01-I-IN, (http://www.csb.gov/assets/document/Hayes_Report.pdf). 


\section{Tables}

Table 1. Comparision of the measurements and the numerical predictions in various groups.

\begin{tabular}{|c|c|c|c|c|c|c|}
\hline \multirow[t]{2}{*}{ Group } & \multirow[t]{2}{*}{ Description } & \multirow[t]{2}{*}{ Configuration } & \multicolumn{2}{|c|}{$\begin{array}{c}\text { Overpressure } \\
\text { (mbar) }\end{array}$} & \multicolumn{2}{|c|}{$\begin{array}{c}\text { Incidence time } \\
(\mathrm{ms})\end{array}$} \\
\hline & & & Exp & LES & Exp & LES \\
\hline \multirow{3}{*}{1} & \multirow{3}{*}{$\begin{array}{l}\text { Baffles are progressively } \\
\text { increased and kept } \\
\text { farthest from ignition end }\end{array}$} & 5 & 80 & 71 & 13.5 & 13.3 \\
\hline & & 2 & 122 & 107 & 11.9 & 11.9 \\
\hline & & 1 & 138 & 125 & 10.3 & 11.0 \\
\hline \multirow{3}{*}{2} & \multirow{3}{*}{$\begin{array}{c}\text { Baffles are progressively } \\
\text { increased from ignition } \\
\text { source }\end{array}$} & 7 & $45^{*}$ & 58 & $11.0^{*}$ & 12.5 \\
\hline & & 4 & 77 & 60 & 9.8 & 10.8 \\
\hline & & 1 & 138 & 125 & 10.3 & 11.0 \\
\hline \multirow{3}{*}{3} & \multirow{3}{*}{$\begin{array}{c}\text { Two baffles are } \\
\text { positioned at different } \\
\text { stations of the chamber }\end{array}$} & 2 & 122 & 107 & 11.9 & 11.9 \\
\hline & & 3 & 87 & 82 & 11.6 & 12.0 \\
\hline & & 4 & 77 & 60 & 9.8 & 10.8 \\
\hline \multirow{3}{*}{4} & \multirow{3}{*}{$\begin{array}{c}\text { Only one baffle } \\
\text { positioned at different } \\
\text { stations of the chamber }\end{array}$} & 5 & 80 & 71 & 13.5 & 13.3 \\
\hline & & 6 & $63^{*}$ & 65 & $10.0^{*}$ & 12.1 \\
\hline & & 7 & $45^{*}$ & 58 & $11.0^{*}$ & 12.5 \\
\hline & ase Configuration & 0 & 34 & 37 & 13.5 & 13.5 \\
\hline
\end{tabular}

* Indicated pressure and time using rich mixture $(\phi=1.1)$ in the same setup having same obstacles at exactly same locations [23].

Table 2. Flamelet regimes and their corresponding times in various configurations of Group 1.

\begin{tabular}{|c|c|c|c|c|}
\hline \multirow{2}{*}{$\mathbf{u}^{\prime} / \mathbf{u}_{\mathbf{L}}$} & \multirow{2}{*}{$\begin{array}{l}\text { Karlovitz } \\
\text { Number } \\
\quad \text { (Ka) }\end{array}$} & \multicolumn{3}{|c|}{ Time (ms) } \\
\hline & & Configuration 1 & Configuration 2 & Configuration 5 \\
\hline $\begin{array}{c}\mathrm{u}^{\prime} / \mathrm{u}_{\mathrm{L}}<1 \text { (Wrinkled } \\
\text { Flamelet Regime) }\end{array}$ & $\mathrm{Ka}<0.8$ & 0 to 2.5 & 0 to 2.5 & 0 to 2.5 \\
\hline $\begin{array}{c}1<\mathrm{u}^{\prime} / \mathrm{u}_{\mathrm{L}}<3 \\
\text { (Corrugated Flamelet } \\
\text { Regime) }\end{array}$ & $0.8<\mathrm{Ka}<1$ & 2.6 to 4.2 & 2.6 to 5.5 & 2.6 to 5.5 \\
\hline $\begin{array}{c}3<\mathrm{u}^{\prime} / \mathrm{u}_{\mathrm{L}}<50 \text { (Thin } \\
\text { Reaction Regime) }\end{array}$ & $1<\mathrm{Ka}<100$ & $\begin{array}{l}4.3 \text { to until flame } \\
\text { exits of the } \\
\text { chamber }\end{array}$ & $\begin{array}{l}5.6 \text { to until the } \\
\text { flame exits the } \\
\text { chamber }\end{array}$ & $\begin{array}{l}6.1 \text { to until the } \\
\text { flame exits the } \\
\text { chamber }\end{array}$ \\
\hline
\end{tabular}




\section{List of Captions for the Figures}

Figure 1: (a) Schematic diagram of the premixed combustion chamber. All dimensions are in $\mathrm{mm}$ (b) Illustration of various flow configurations employed (Not to scale) (c) Illustration of the computational domain. Combustion chamber and other obstacles are superimposed over grid resolution.

Figure 2: (a) Pressure-time histories of five different runs of configuration1. Average pressure-time trend is also shown in black solid line. (b) Velocity-time histories of 50 experiments together with their mean and fitted least-square polynomial of configuration 3 (reprinted with permission from author of reference 31 ).

Figure 3: Time histories of overpressure and flame position for configuration 0 .

Figure 4: Series of flame images at 6.0, 12.0, 12.5, 13.0, 13.5, 14.0, 14.5, 15.0, $15.5 \mathrm{~ms}$ respectively after ignition (a) LES (b) Experimental video images (false colourised). Horizontal lines at top and bottom of the chamber seen in video images are draw bolts used to hold the test facility.

Figure 5: Time histories of mean velocities and their rms fluctuations for configuration 0 at the middle of the chamber and half-way up the side of the square obstacle i.e. $102 \mathrm{~mm}$ from the base and $16 \mathrm{~mm}$ from the central axis. The scale on left hand side is for mean velocity and on right hand side is for rms fluctuations. (a) axial velocity (b) radial velocity.

Figure 6: Variation of peak overpressure and its time of incidence compared from LES vs. Experiments for four groups. (a) Group 1 (b) Group 2 (c) Group 3 (d) Group 4, experimental measurements of configuration $6 \& 7$ are from Hall et al. [23].

Figure 7: Comparison of predicted (solid line) and measured (dashed line with symbols) time traces of Group 1 (a) overpressure (b) flame position.

Figure 8: Comparisons between predicted (solid line) and measured (dashed line with symbols) (a) flame speed (b) flame acceleration vs. axial distance. The location of baffle stations (S1, S2 and S3) and the square solid obstacle are shown.

Figure 9: Predicted flame structure from three configurations at 6, 8, 10, 11.5 and $12.0 \mathrm{~ms}$ after ignition (a) Configuration 5 (b) Configuration 2 (c) Configuration 1

Figure 10: (a) Unresolved FSD against normalised velocity fluctuations for three configurations (b) Numerical estimates from three configurations are fitted in the turbulent premixed combustion regime reported by Peters [34]. Here $L_{f}$ is the calculated strained laminar flame thickness and this is different from the unstrained laminar flame thickness, $L_{f 0}$ which is a specified input parameter $\left(L_{f 0}=0.3 \mathrm{~mm}\right)$. (c) Time derivatives of resolved and unresolved flame areas against averaged Karlovitz number in configuration 1.

Figure 11: Comparison of predicted (solid line) and measured (dashed line with symbols) time traces of Group 3 (a) overpressure (b) flame position.

Figure 12: Comparisons between predicted (solid line) and measured (dashed line with square symbols) (a) flame speed (b) flame acceleration vs. axial distance. The location of baffles (S1, S2 and S3) and the square solid obstacle are shown. 
Figure 13: Predicted and video captured (false colourised) flame structure from three configurations of Group 3 at 6, 8, 10,11.5 and $12.0 \mathrm{~ms}$ after ignition. Horizontal lines at top and bottom of the chamber seen in video images are draw bolts used to hold the test facility. (a) Configuration 2 (b) Configuration 3 (c) Configuration 4

Figure 14: Time histories of mean velocities and their rms fluctuations of various configurations in Group 3 at the middle of the chamber and half-way up the side of the square obstacle i.e. $102 \mathrm{~mm}$ from the base and $16 \mathrm{~mm}$ from the central axis. The scale on left hand side is for mean velocity and on right hand side is for rms fluctuations. Experimental measurements are obtained from Hall [31] (a) Configuration 2 (b) Configuration 3 (c) Configuration 4

Figure 15: Flame characteristics of Group 4 (a) Time traces of overpressure (b) Time traces of flame position.

Figure 16: Snapshots of reaction rate contours at 6.0, 8.0, 10.0, 12.0 and $13 \mathrm{~ms}$ in various configurations of Group 4 (a) Configuration 5 (b) Configuration 6 (c) Configuration 7. 
Figures

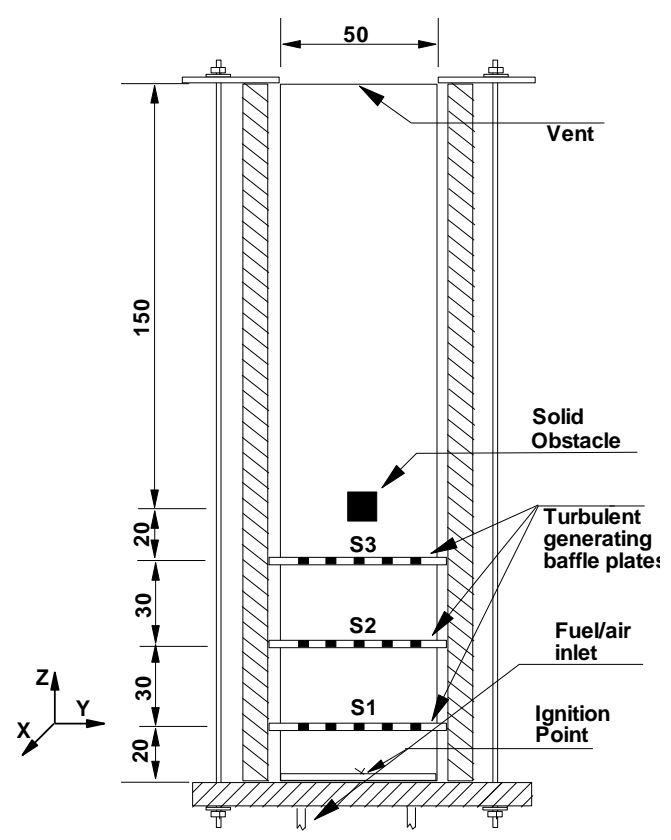

(a)
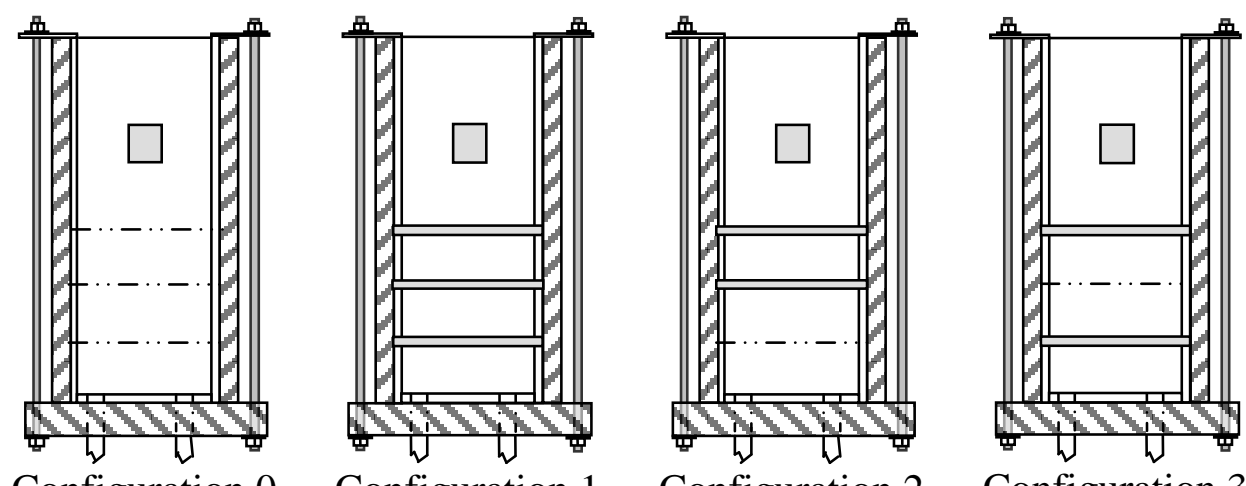

Configuration 0
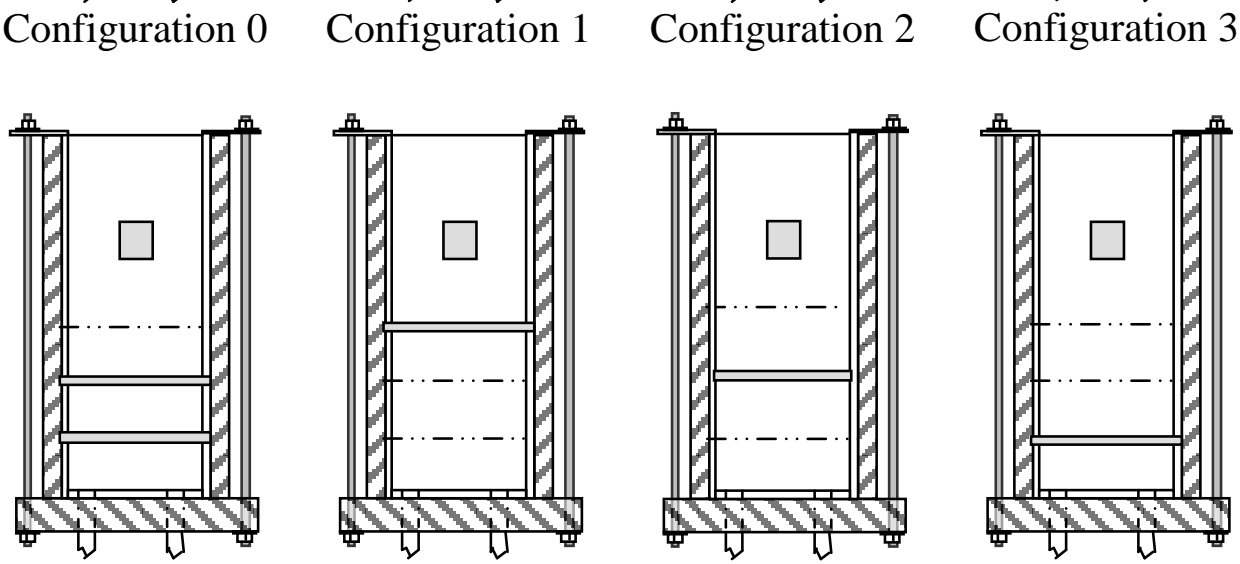

Configuration 4

Configuration 6

(b)

Figure 1 


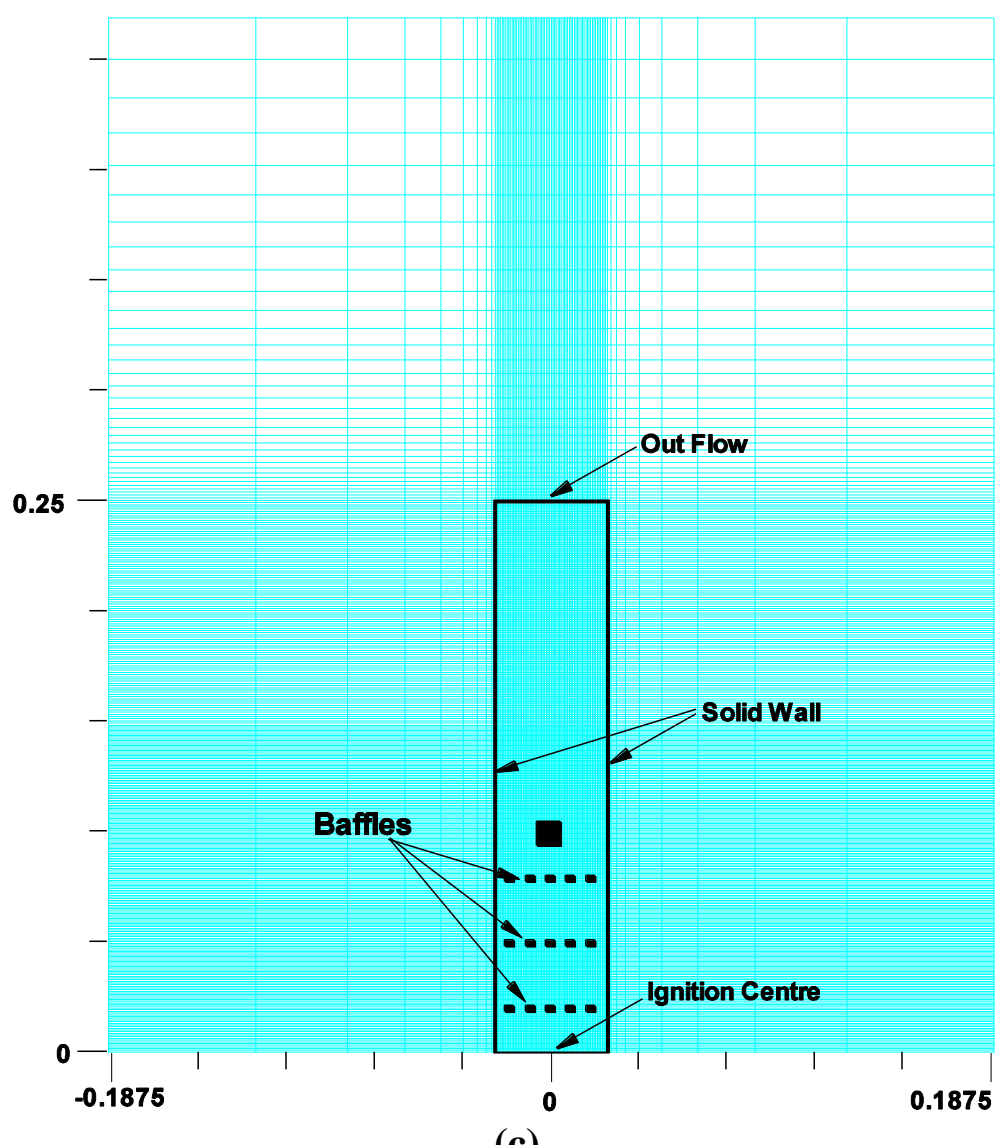

(c)

Figure 1 


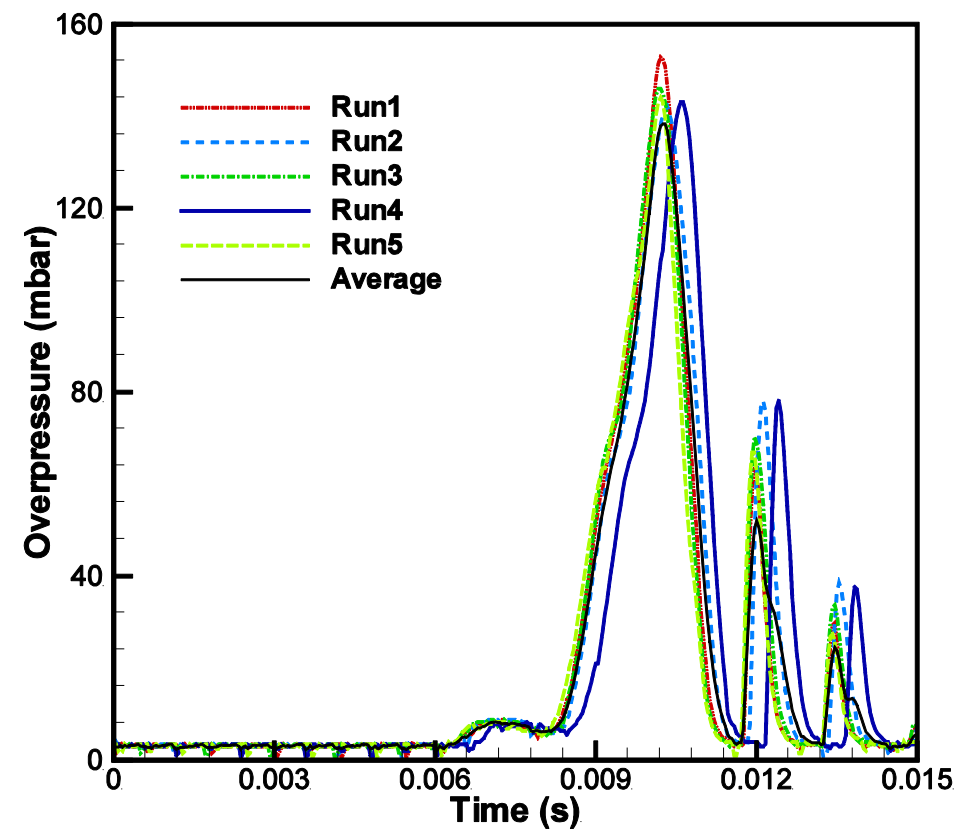

(a)

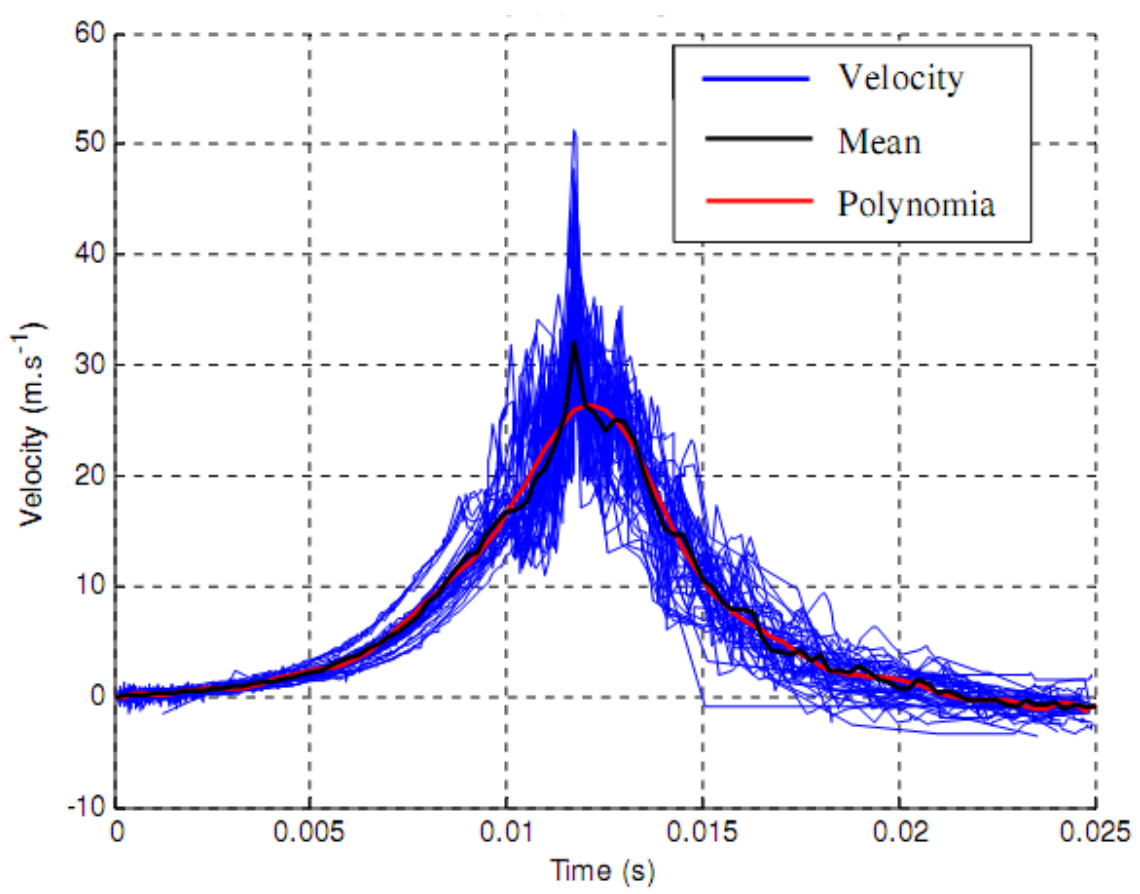

(b)

Figure 2 


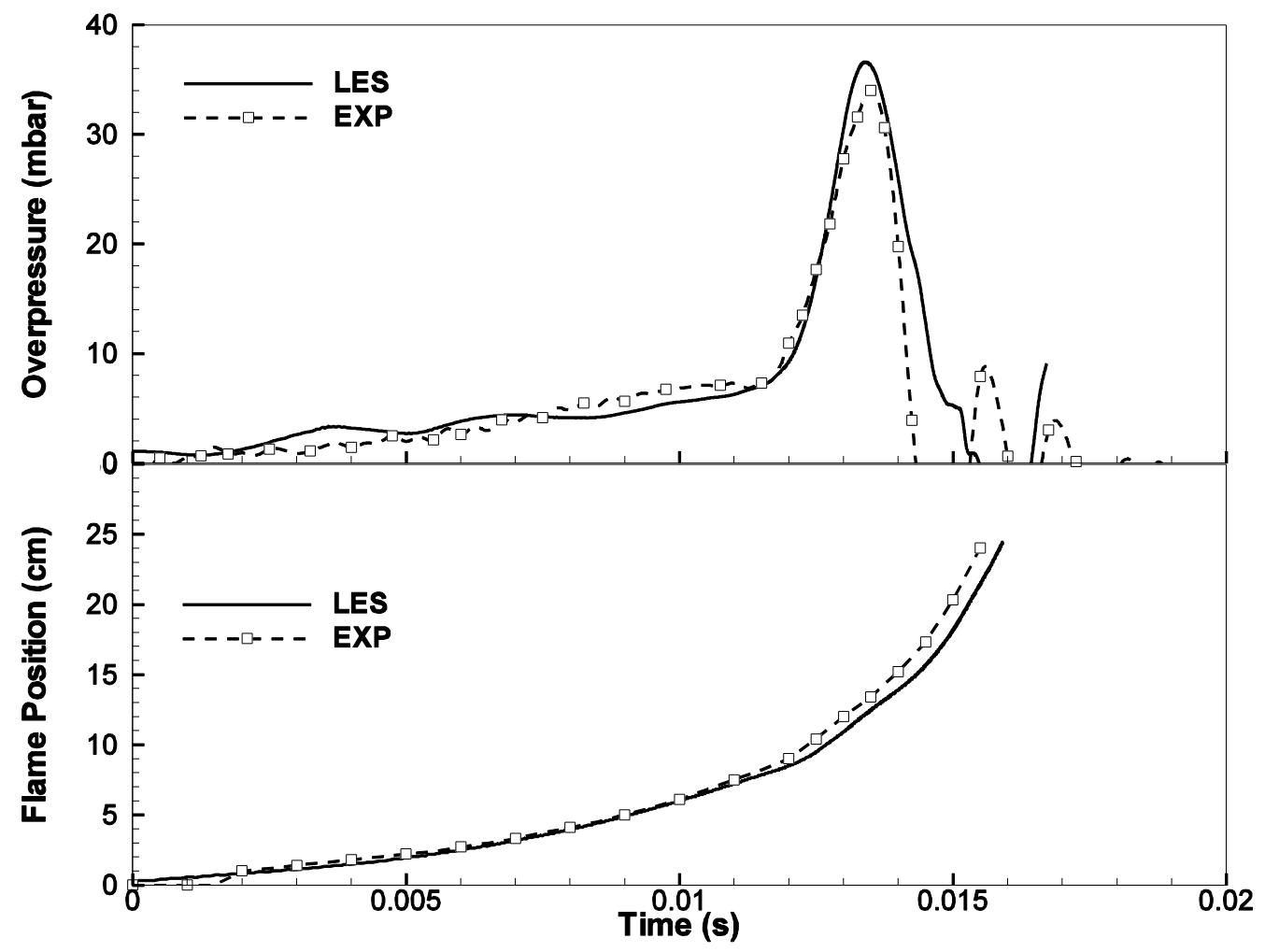

Figure 3 


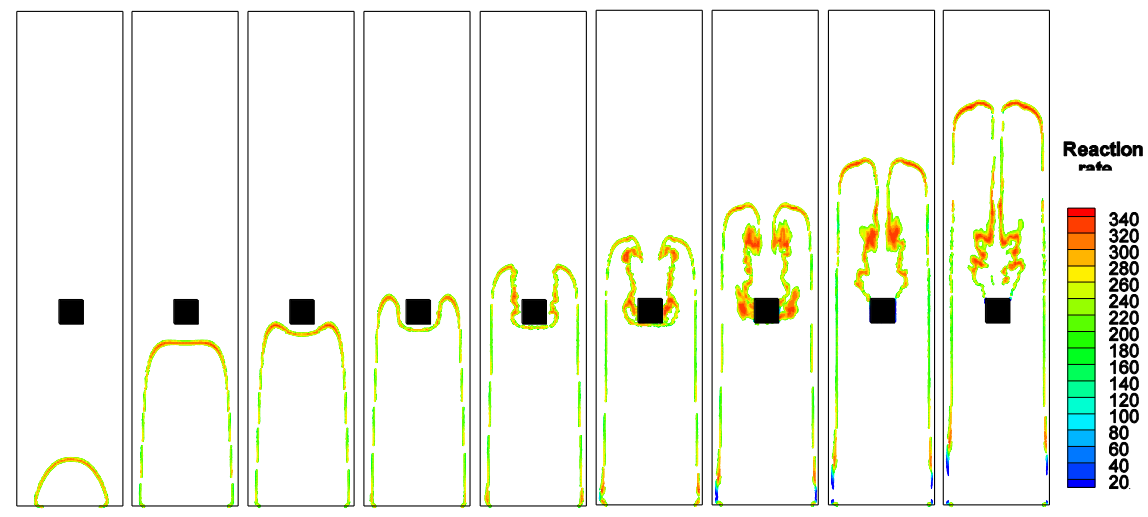

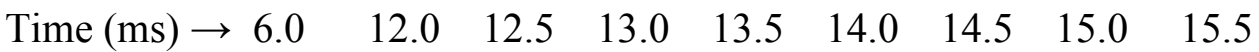

(a)

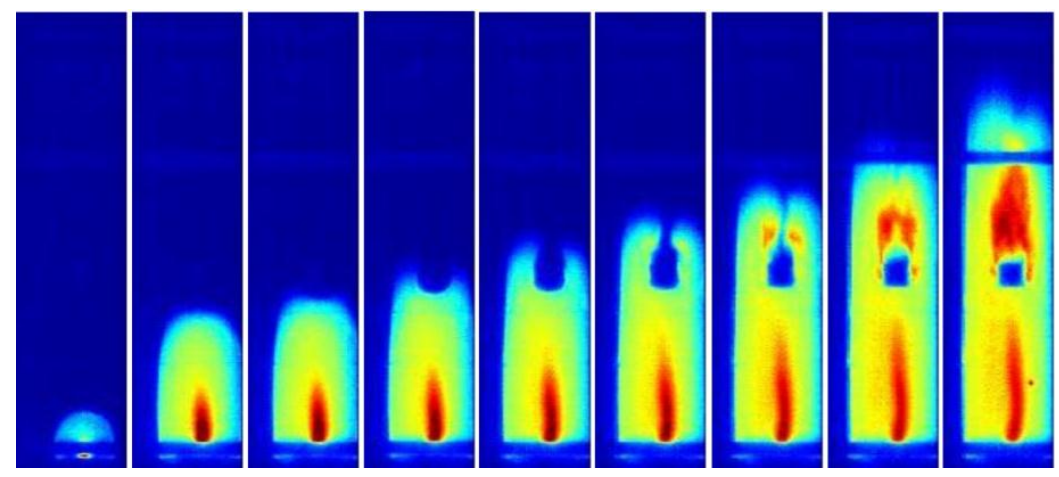

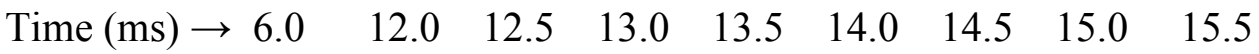

(b)

Figure 4 


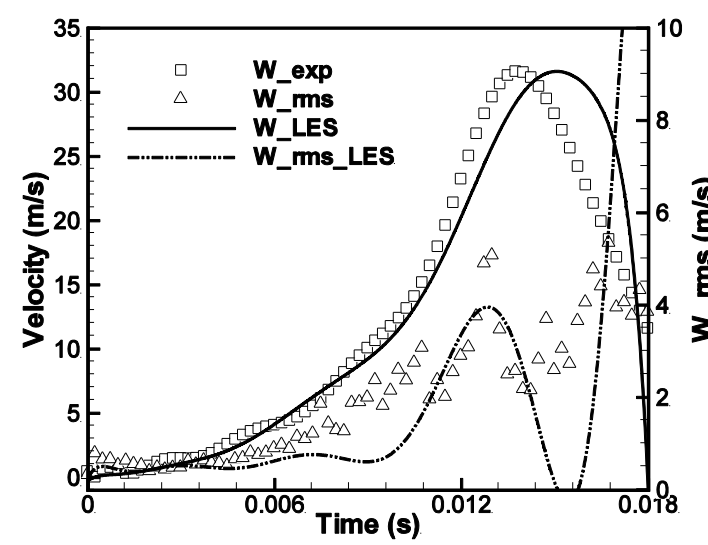

(a)

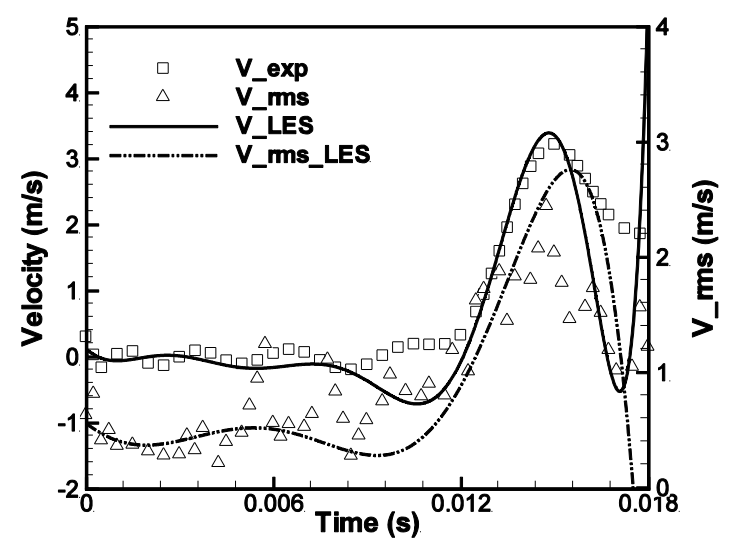

(b)

Figure 5 

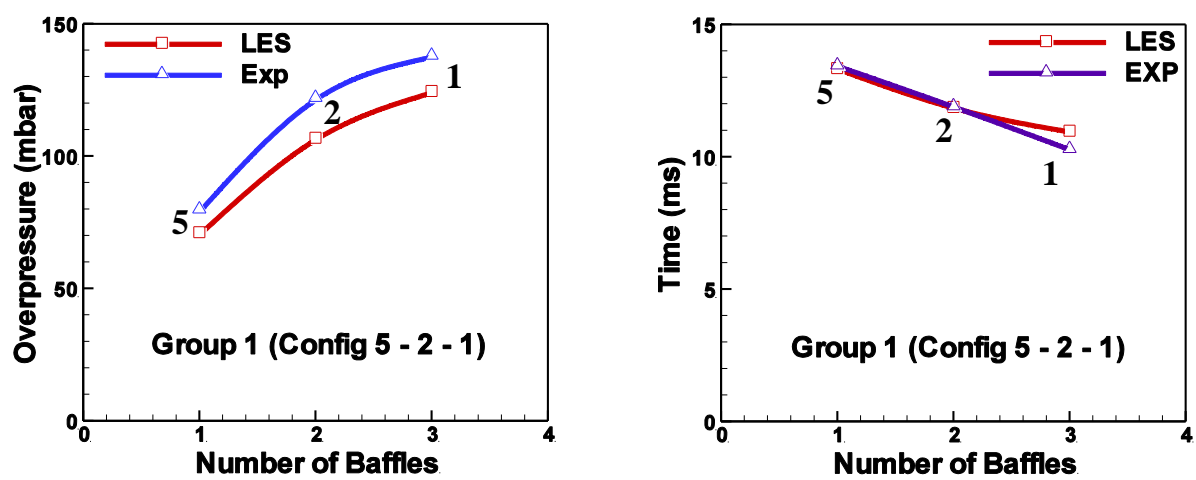

(a) Group 1
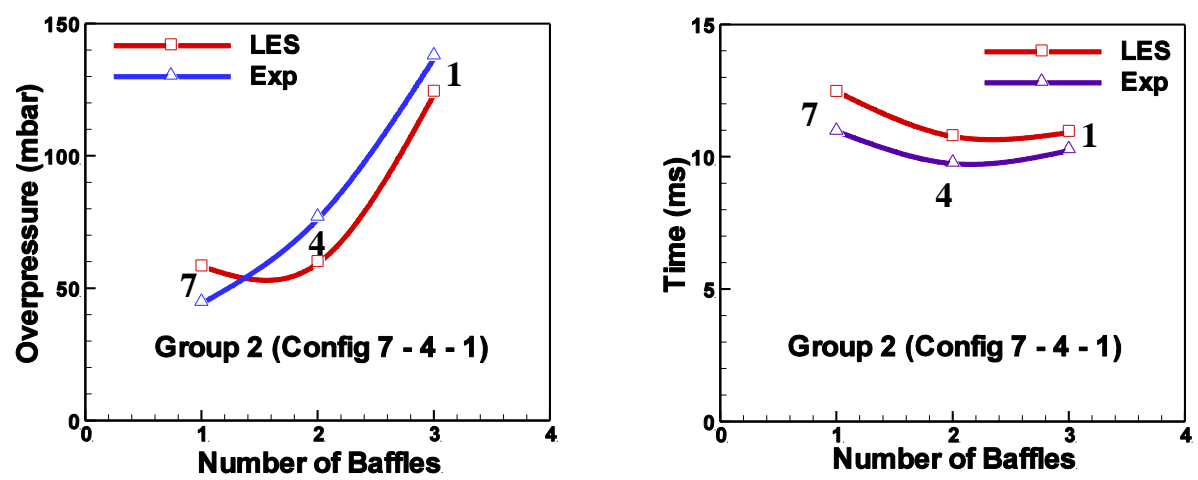

(b) Group 2
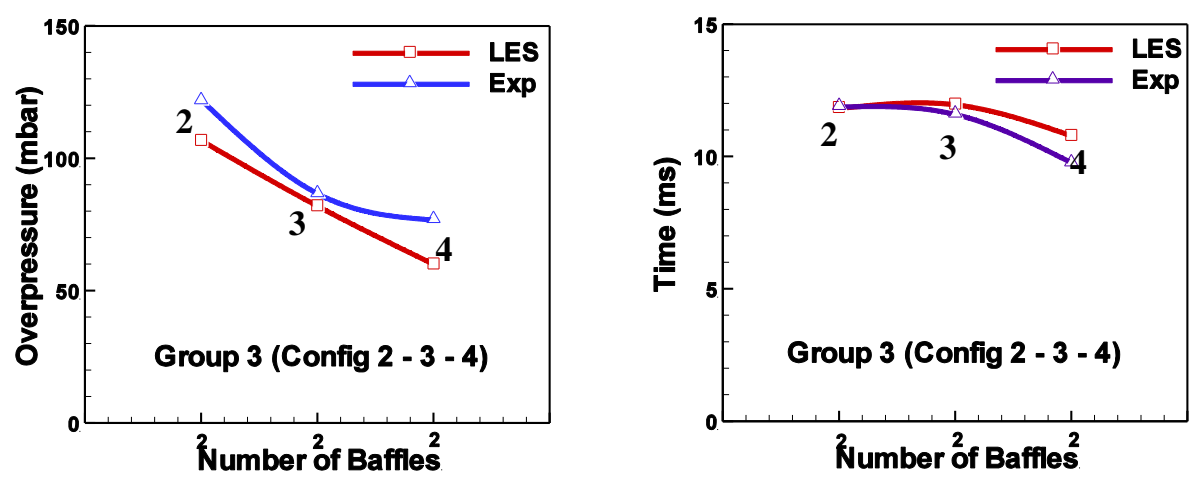

(c) Group 3
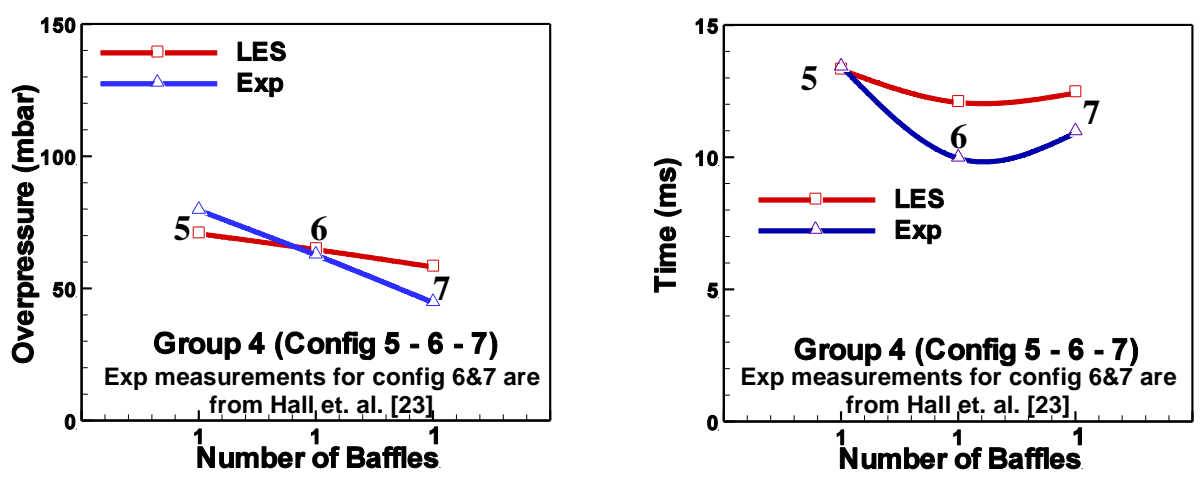

(d) Group 4

Figure 6 


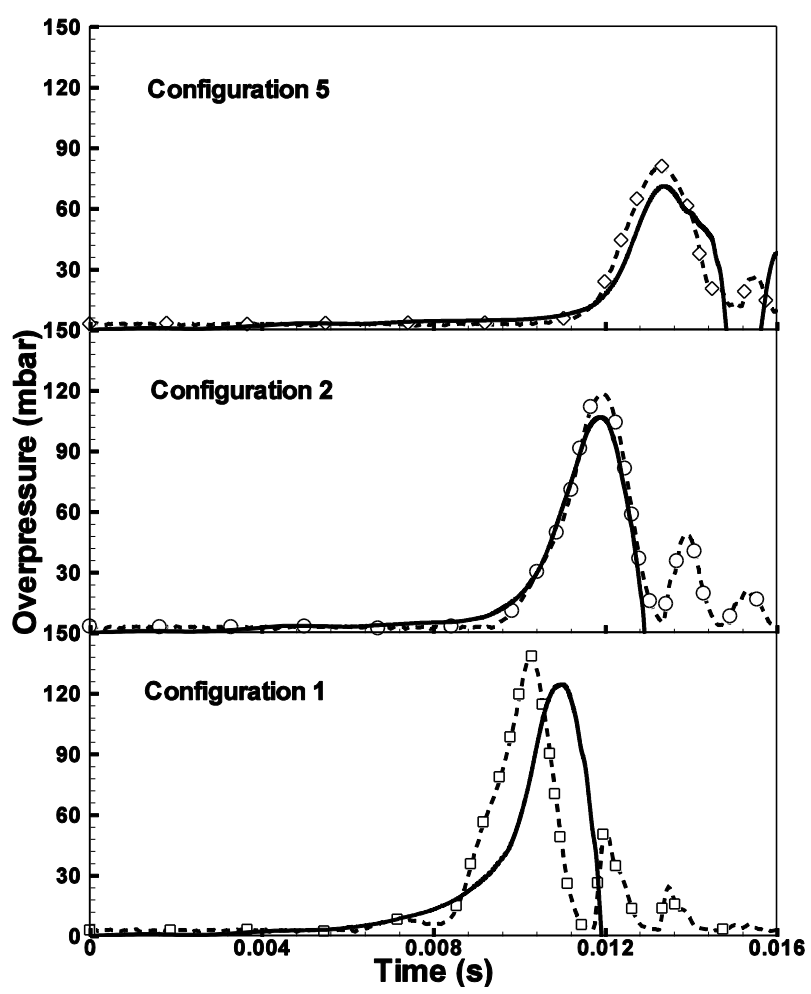

(a)

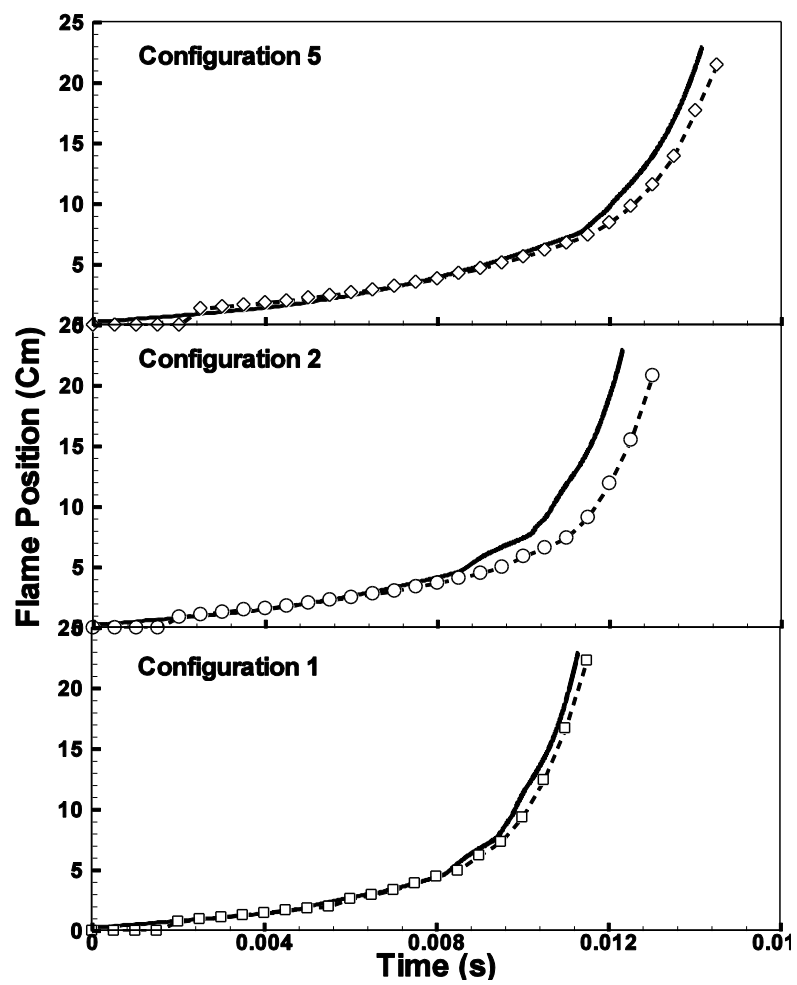

(b)

Figure 7 


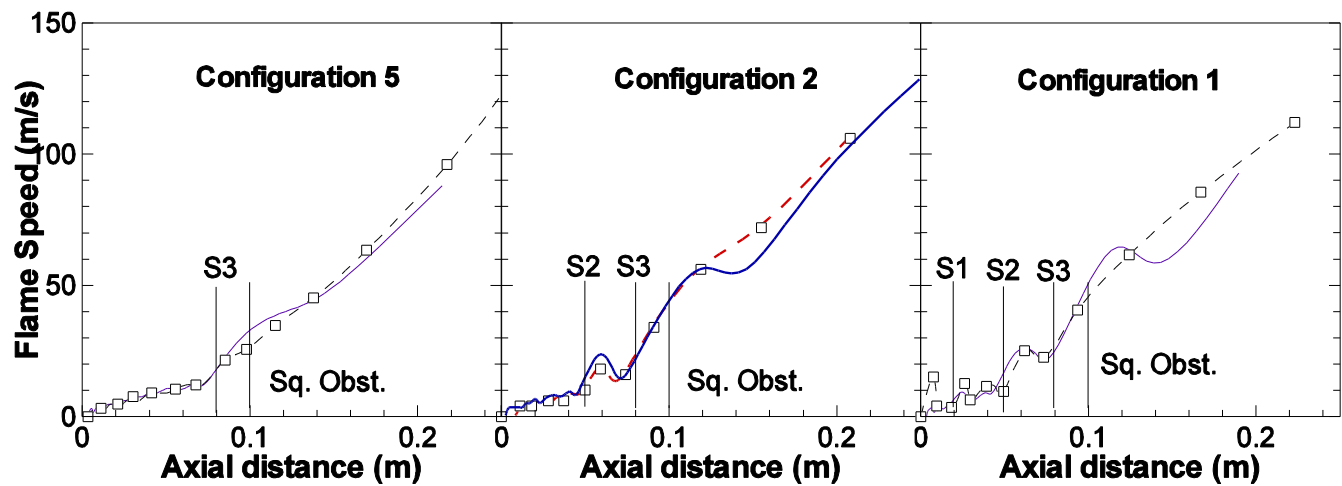

(a)

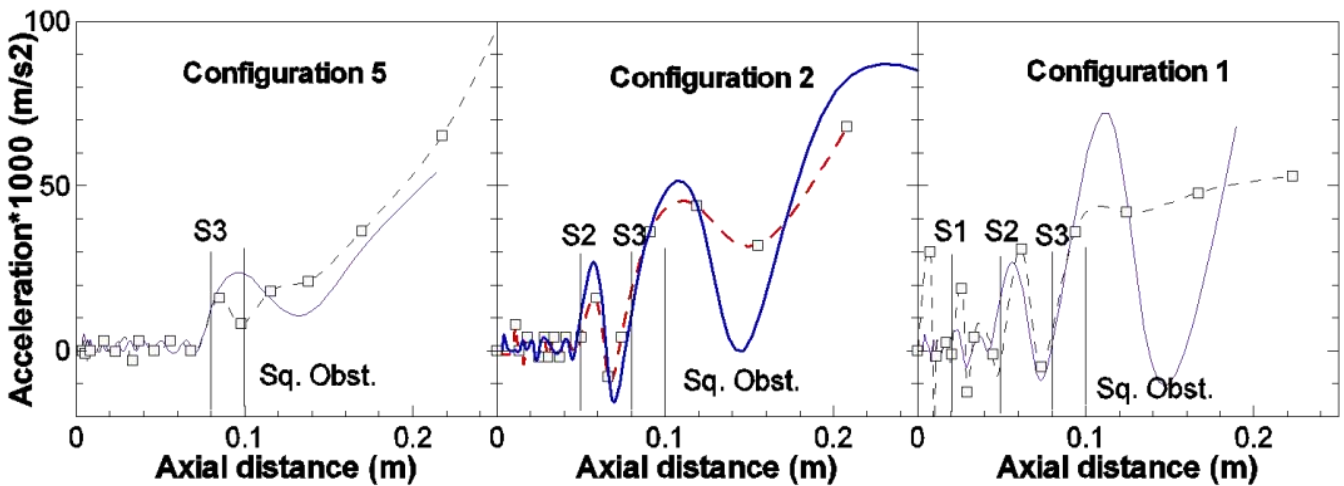

(b)

\section{Figure 8}




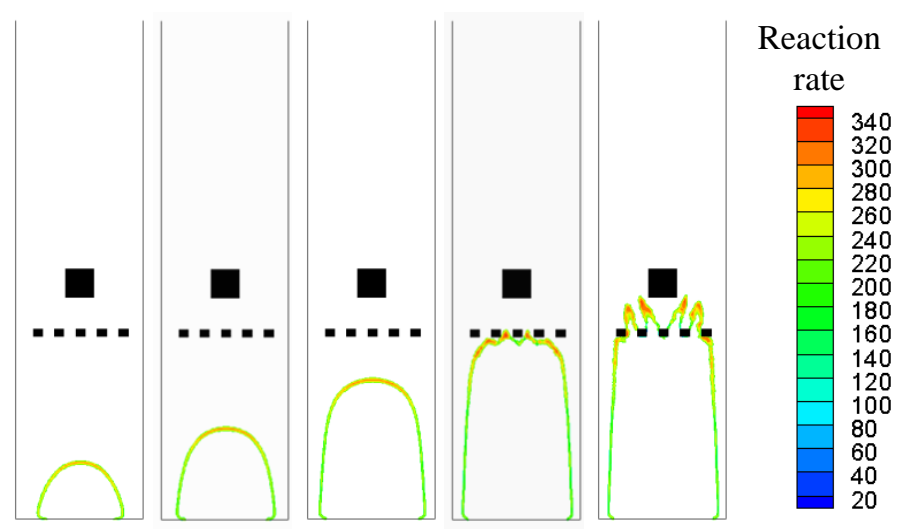

$\begin{array}{llllll}\text { Time (ms) } & 6.0 & 8.0 & 10.0 & 11.5 & 12.0\end{array}$

(a) Configuration 5

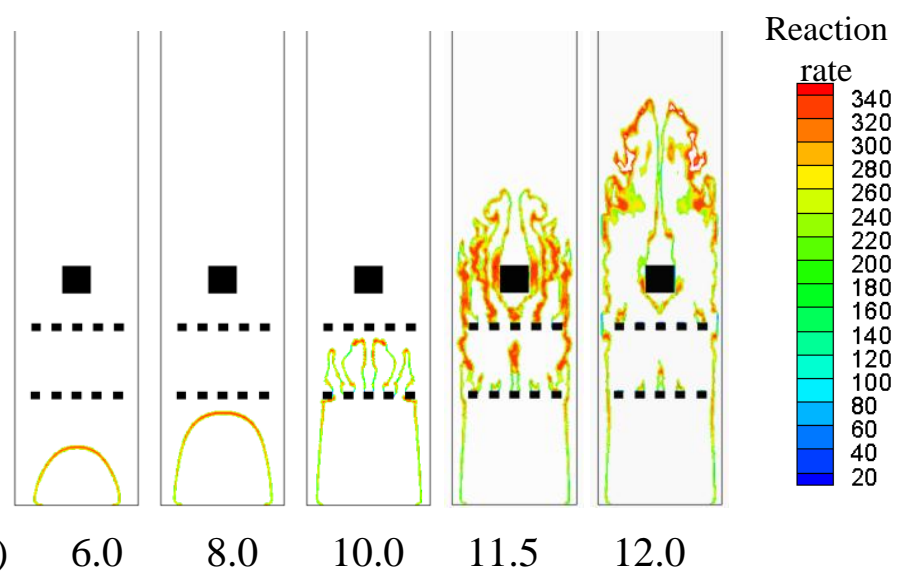

(b) Configuration 2
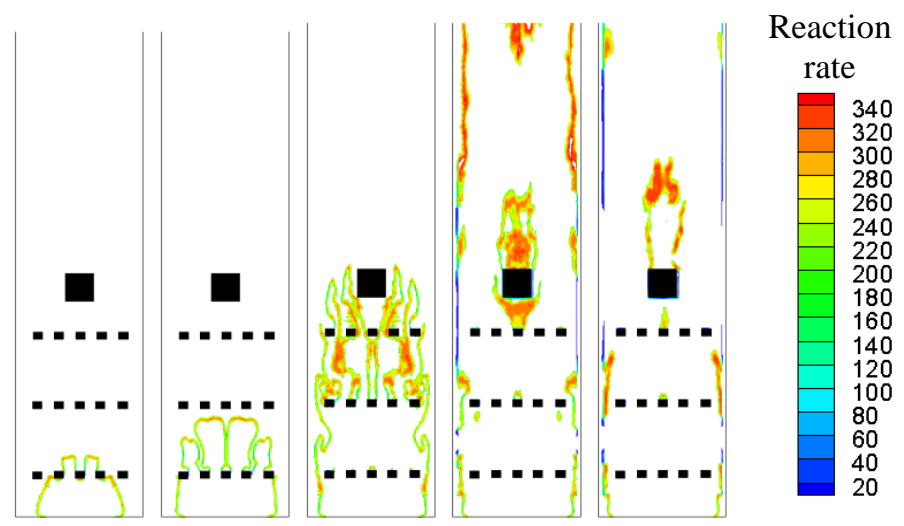

Time (ms)

$$
6.0 \quad 8.0
$$

10.0

$11.5 \quad 12.0$

(c) Configuration 1

\section{Figure 9}




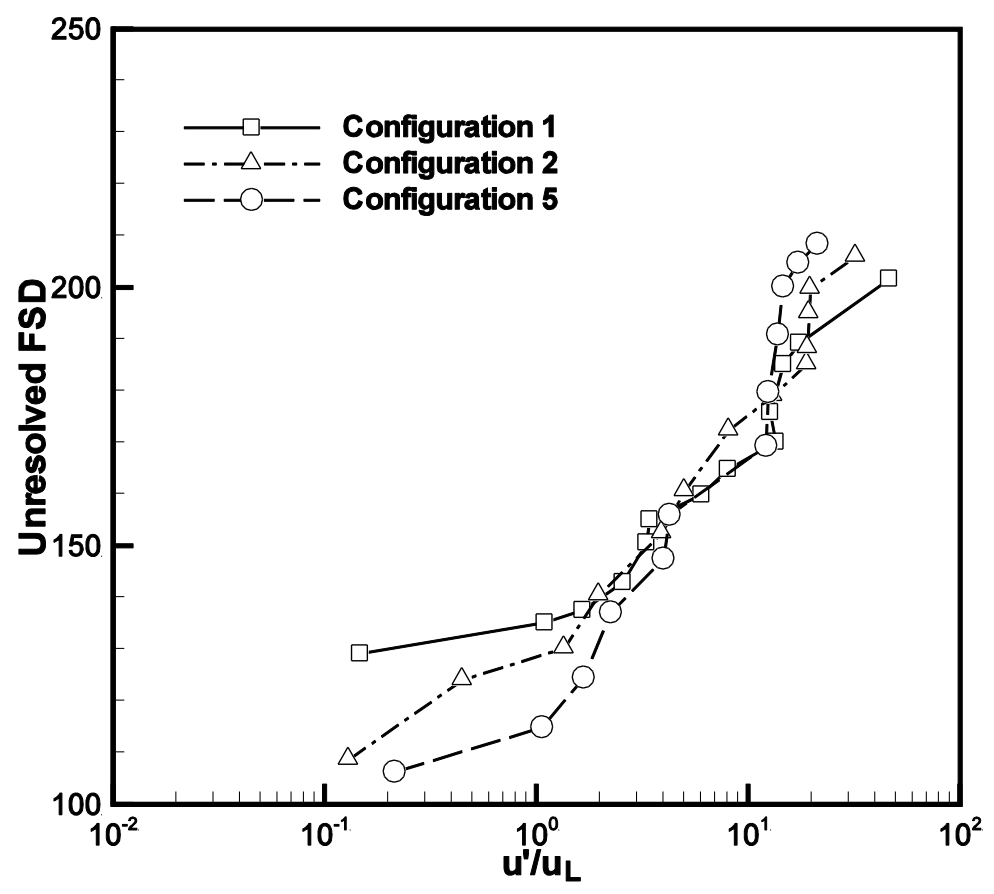

(a)

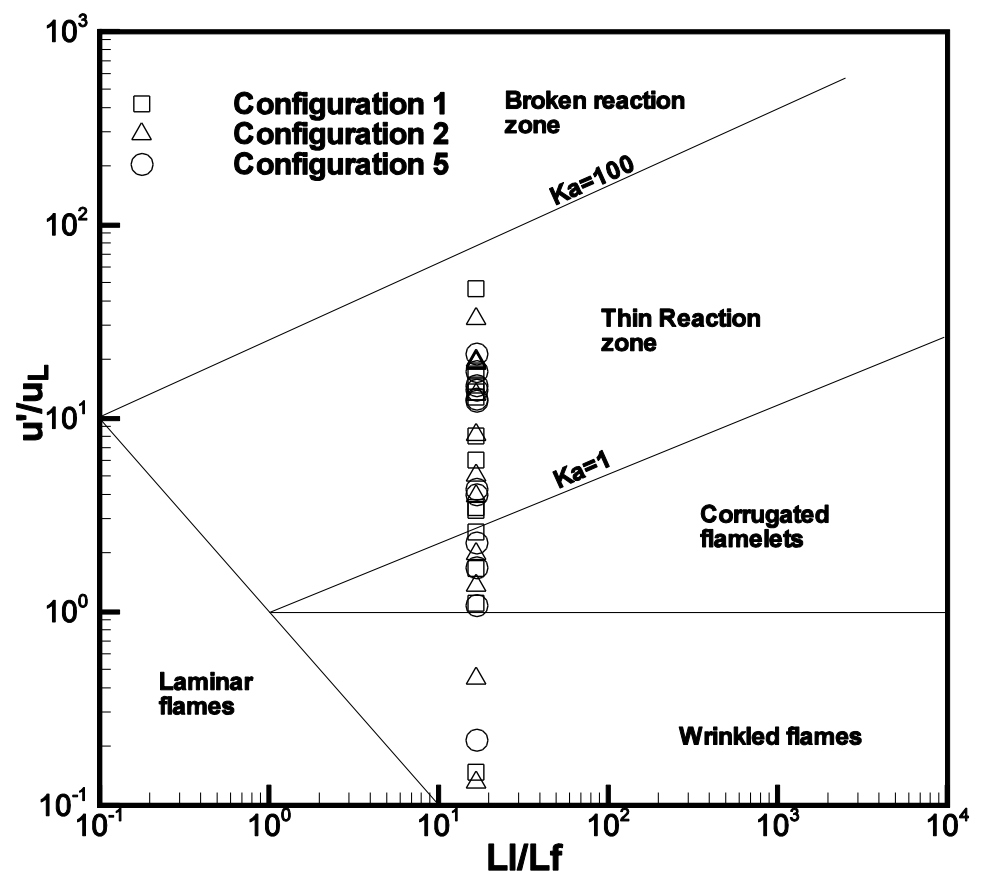

(b)

Figure 10 


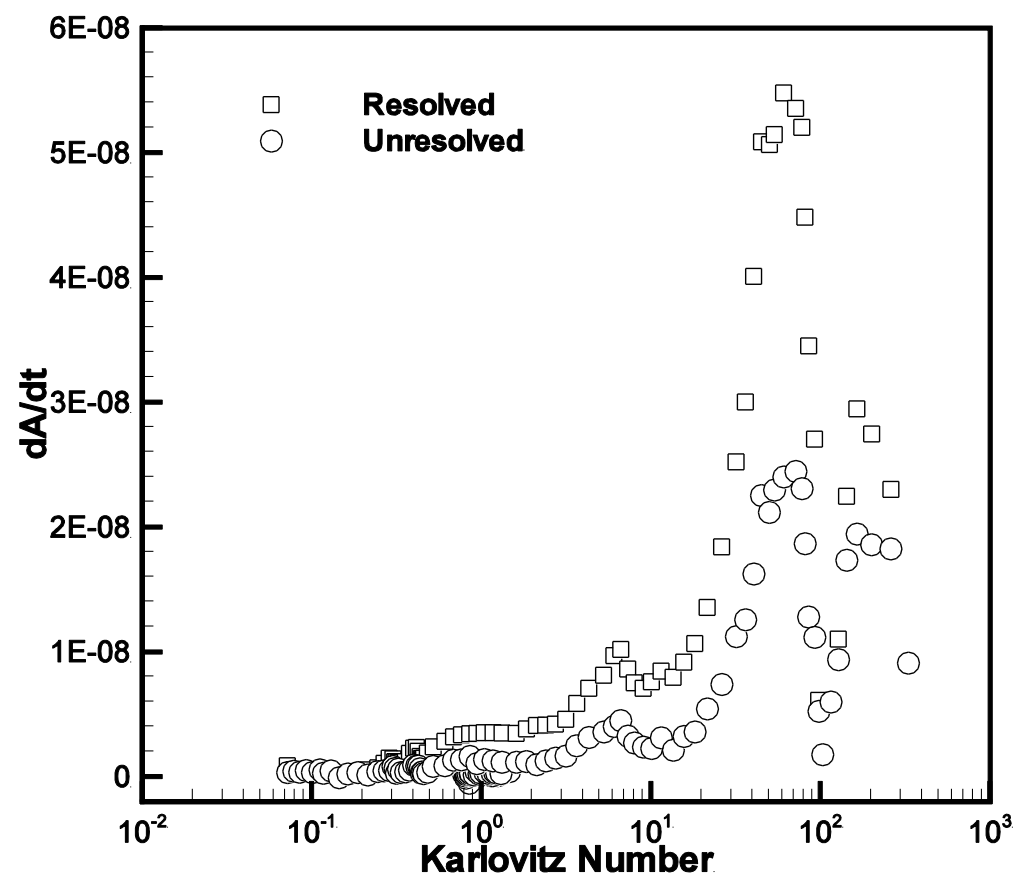

(c)

Figure 10 


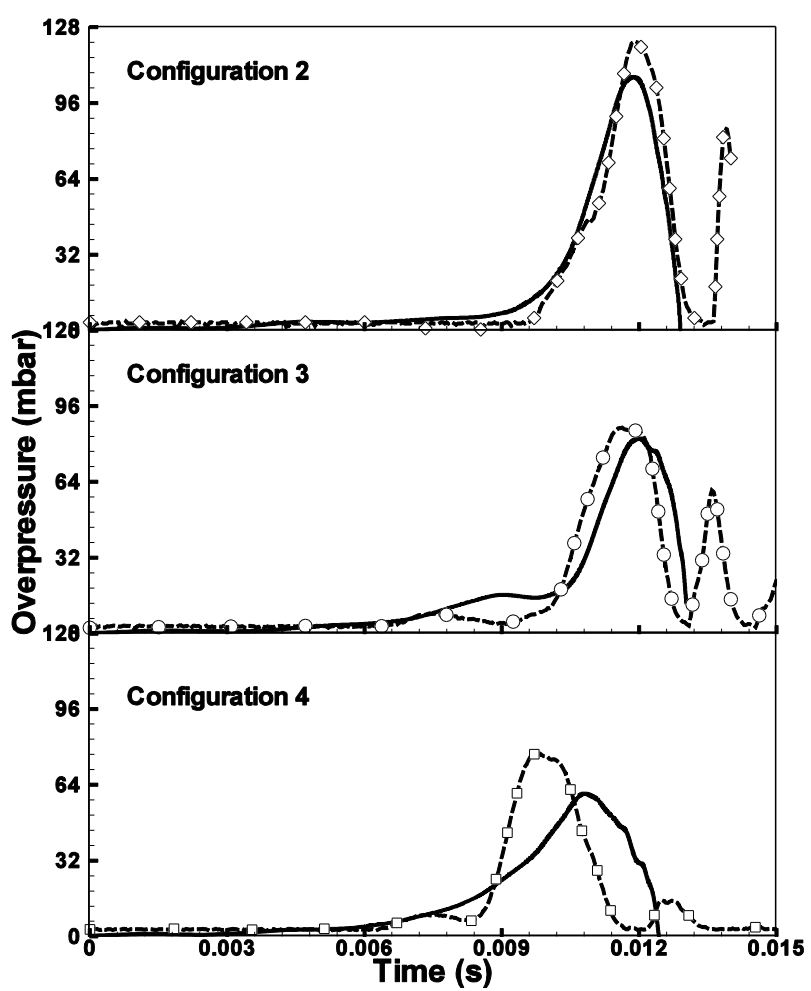

(a)

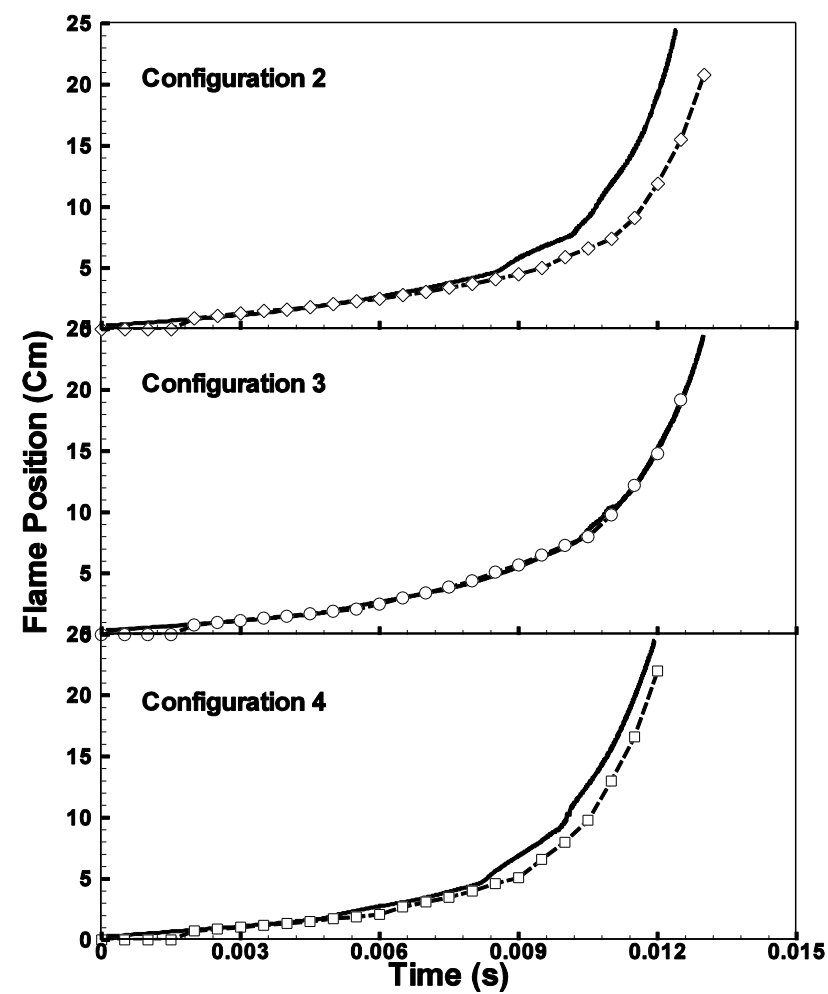

(b)

Figure 11 


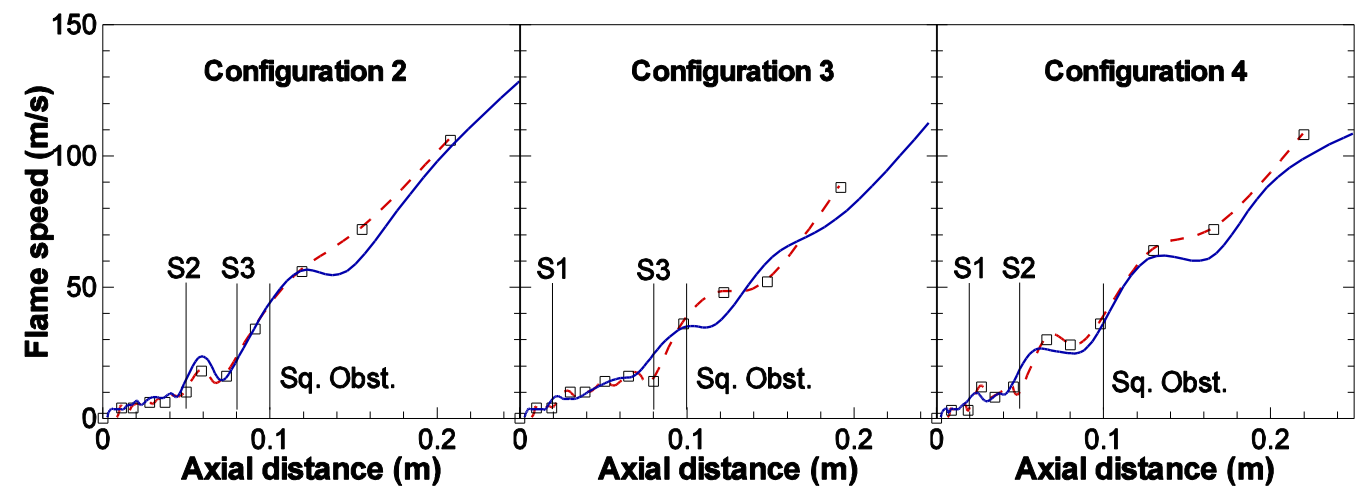

(a)

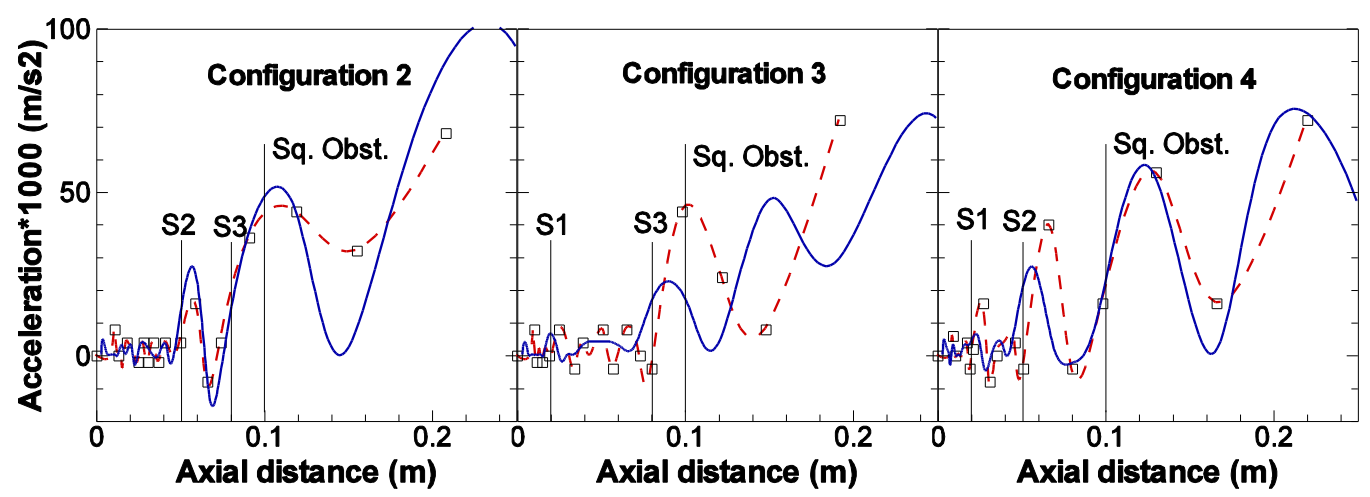

(b)

Figure 12 

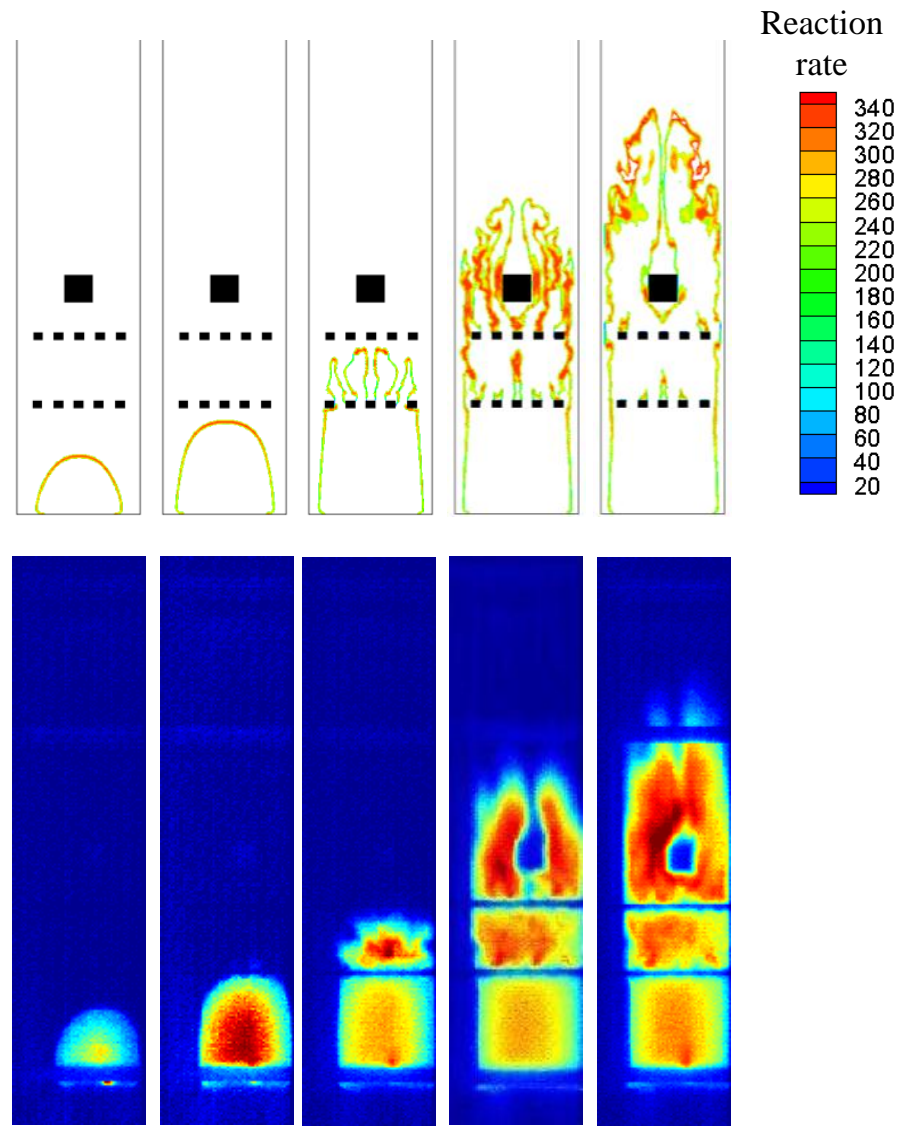

Time (ms)

6.0

8.0

10.0

11.5

12.0

Configuration 2

Figure 13a 


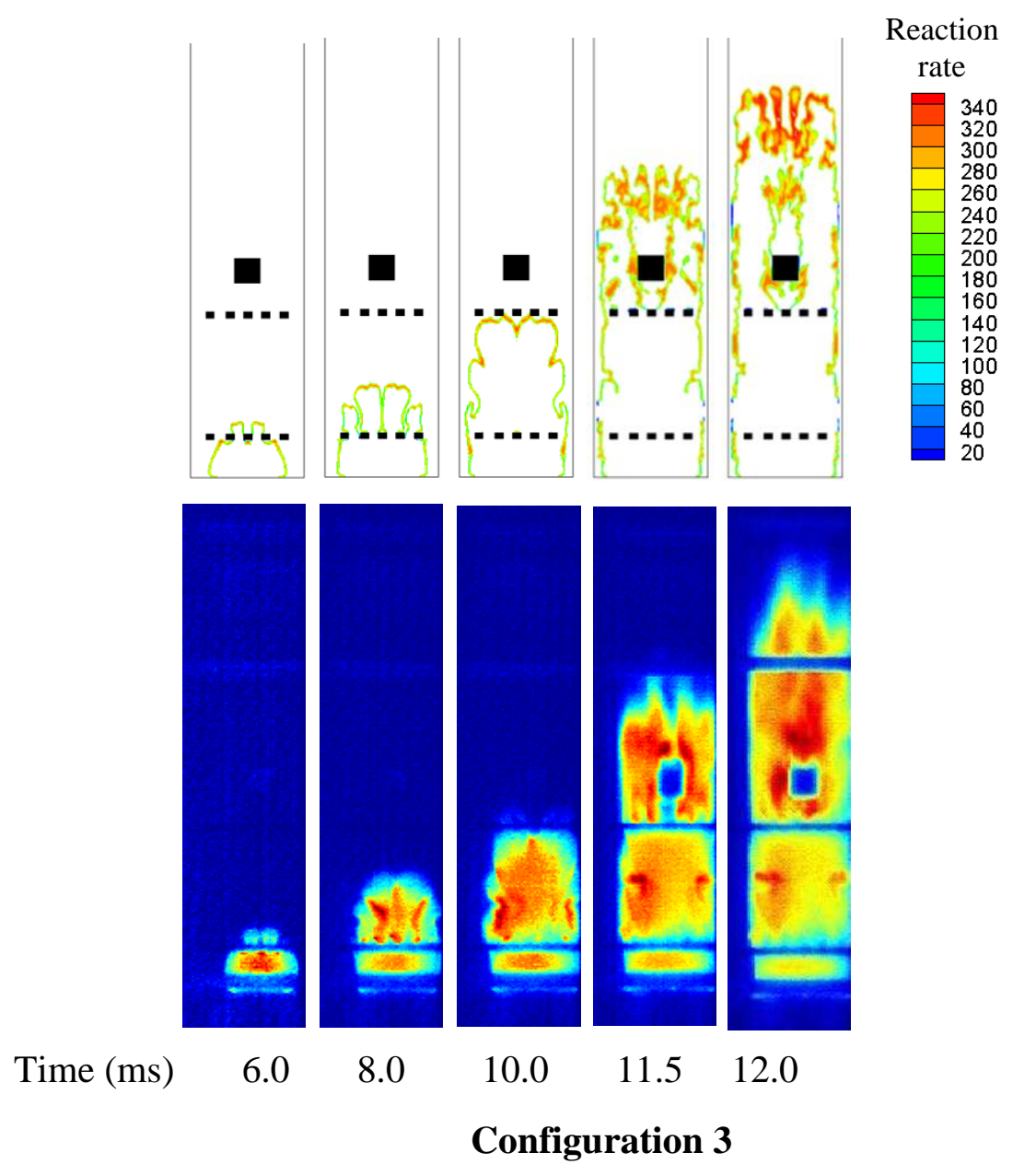

Figure 13b 

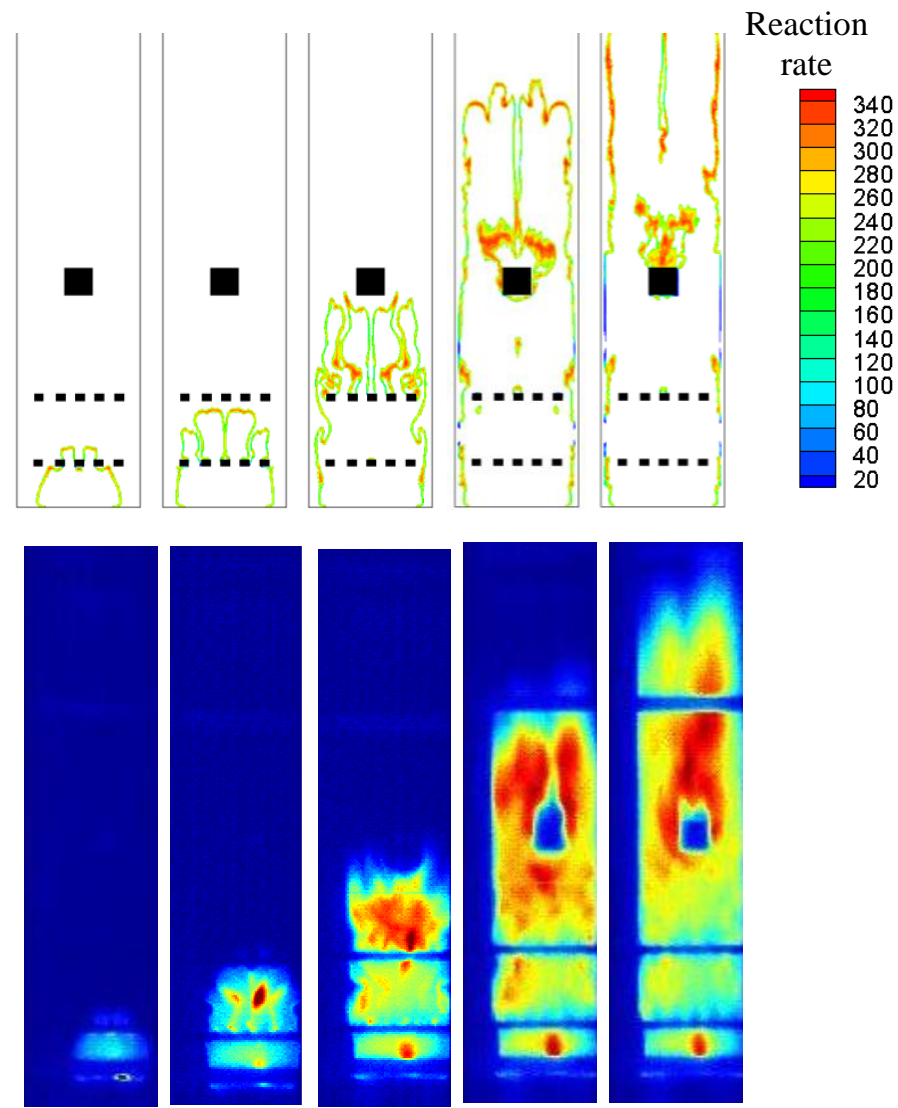

Time (ms)

6.0

8.0

10.0

11.5

12.0

\section{Configuration 4}

Figure 13c 

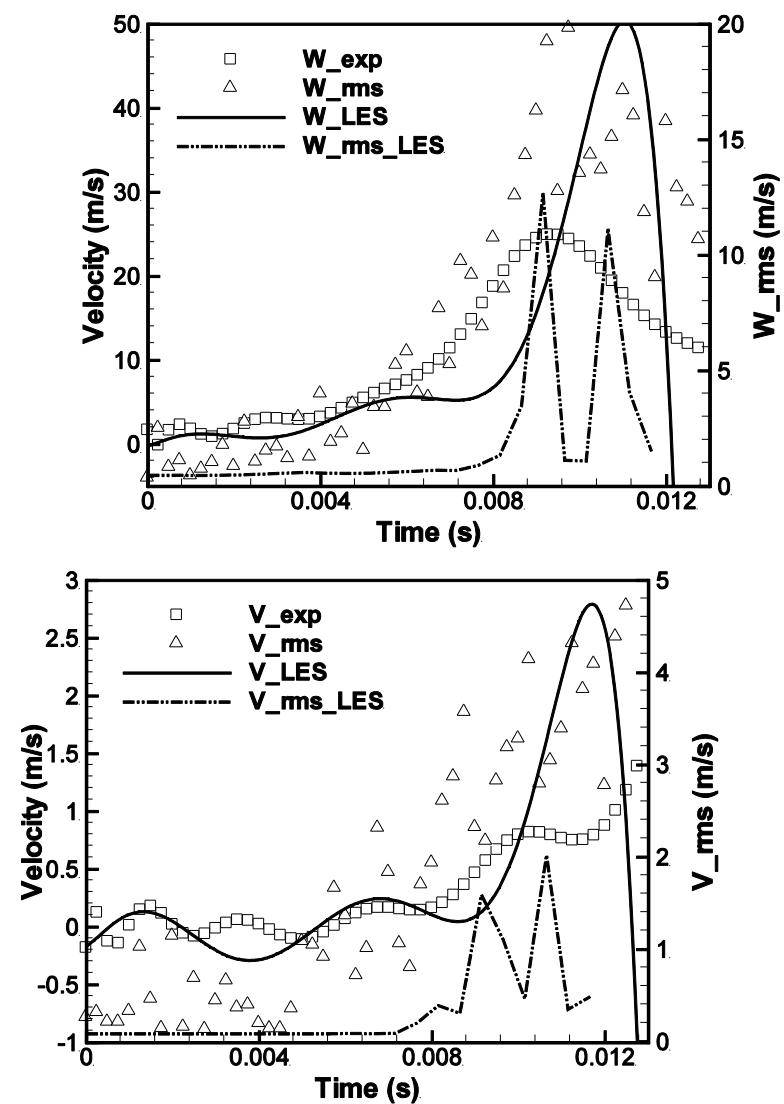

(a) Configuration 2
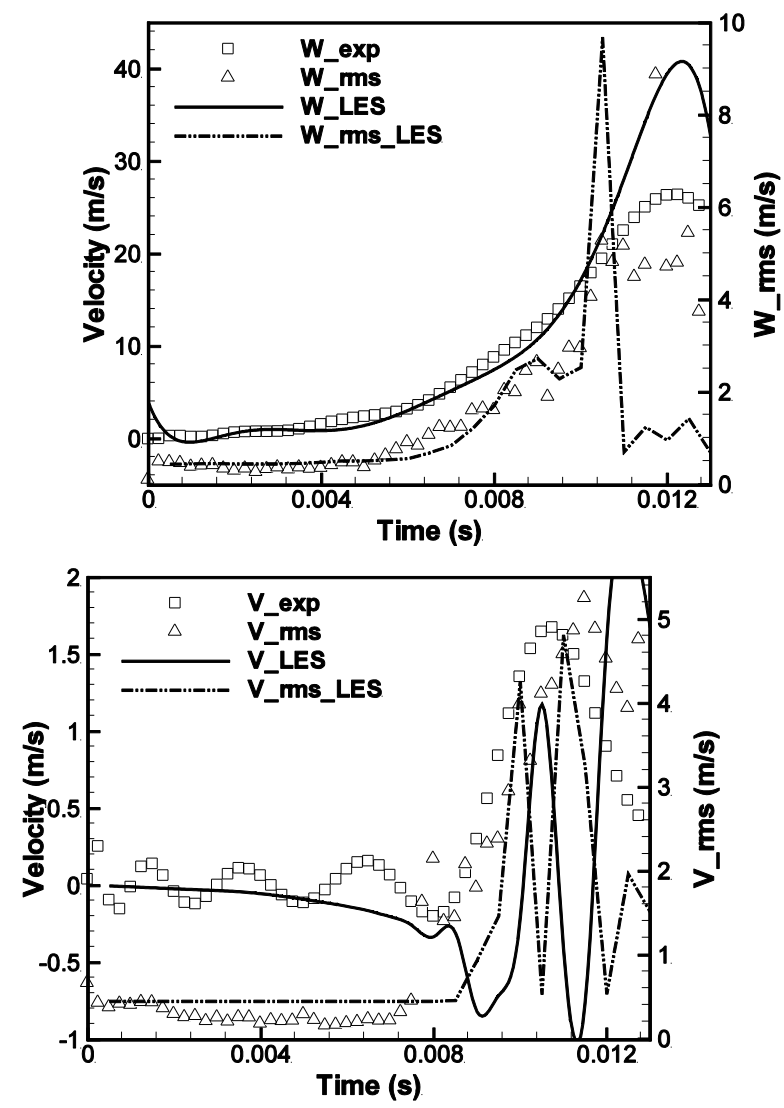

(b) Configuration 3 

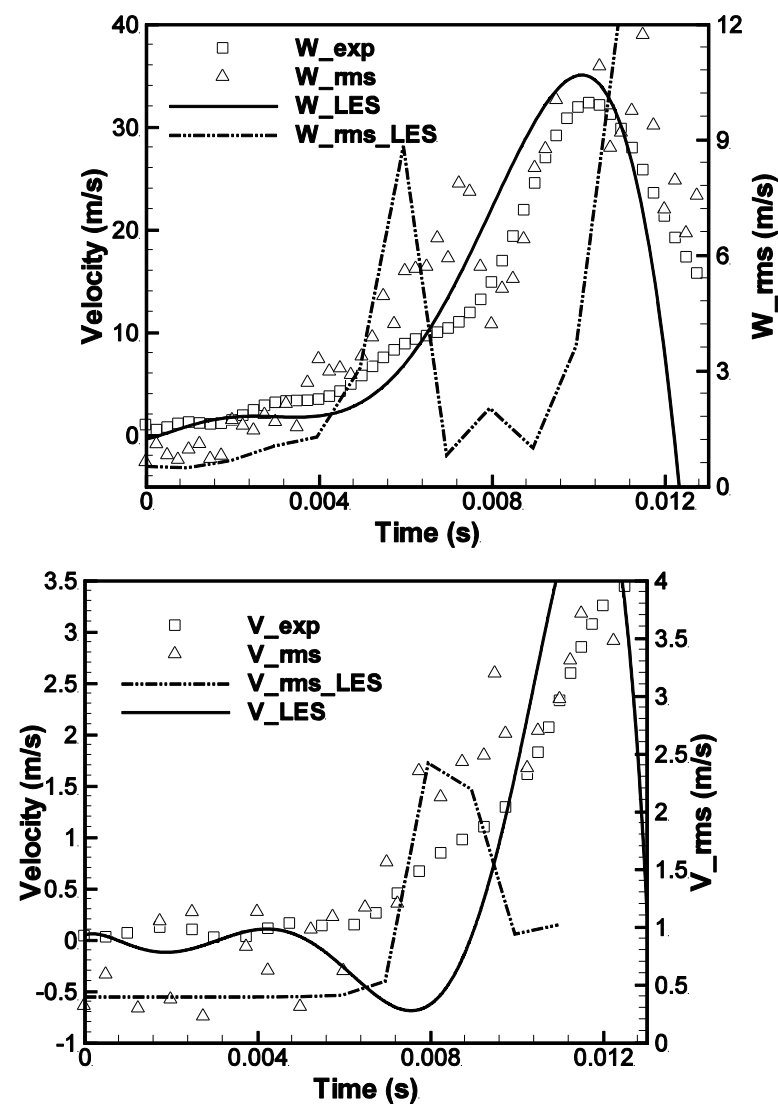

(c) Configuration 4

Figure 14 


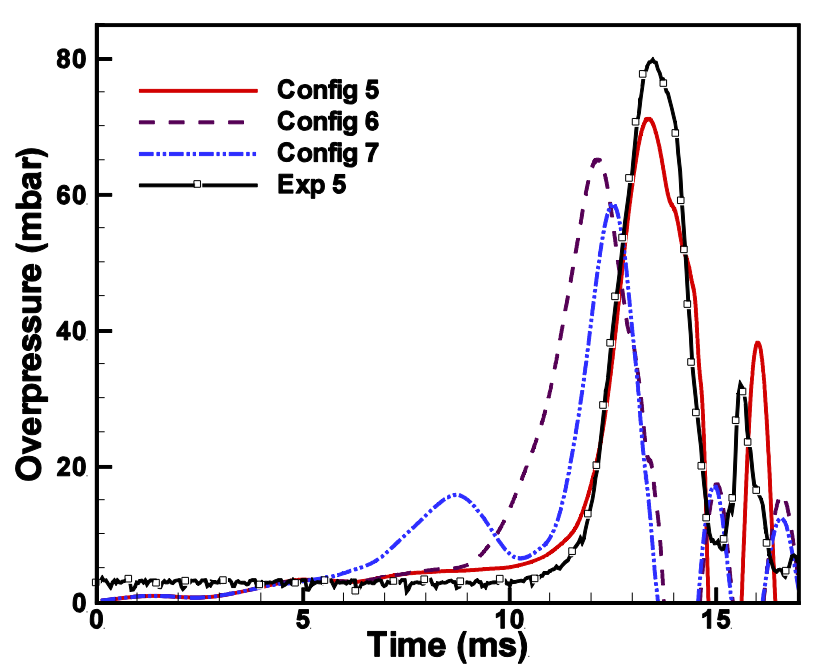

(a)

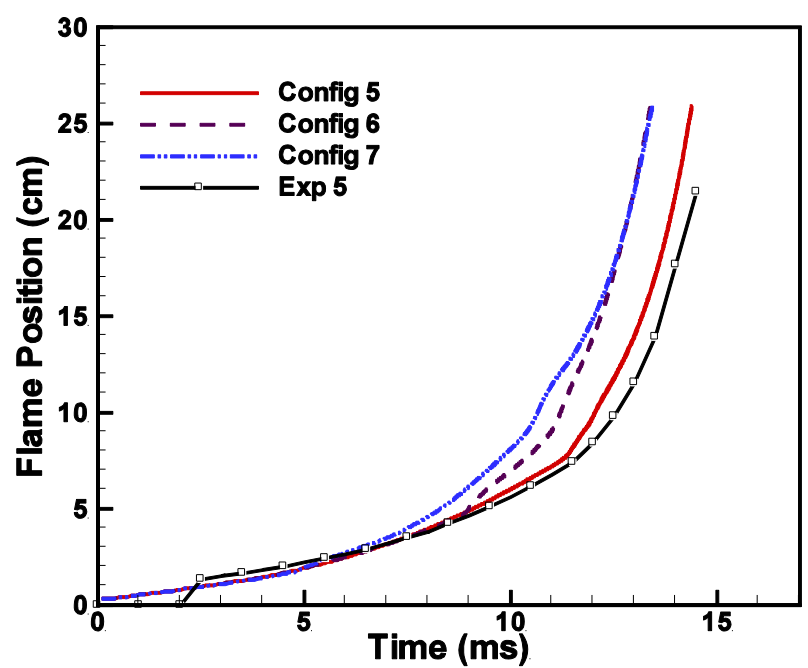

(b)

Figure 15 


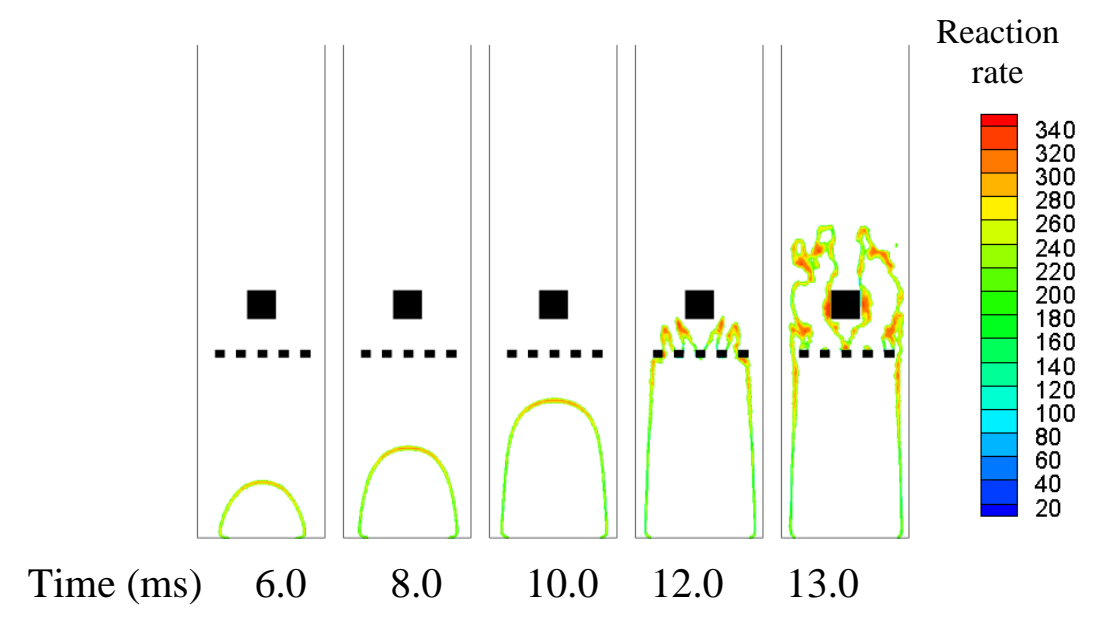

(a) Configuration 5

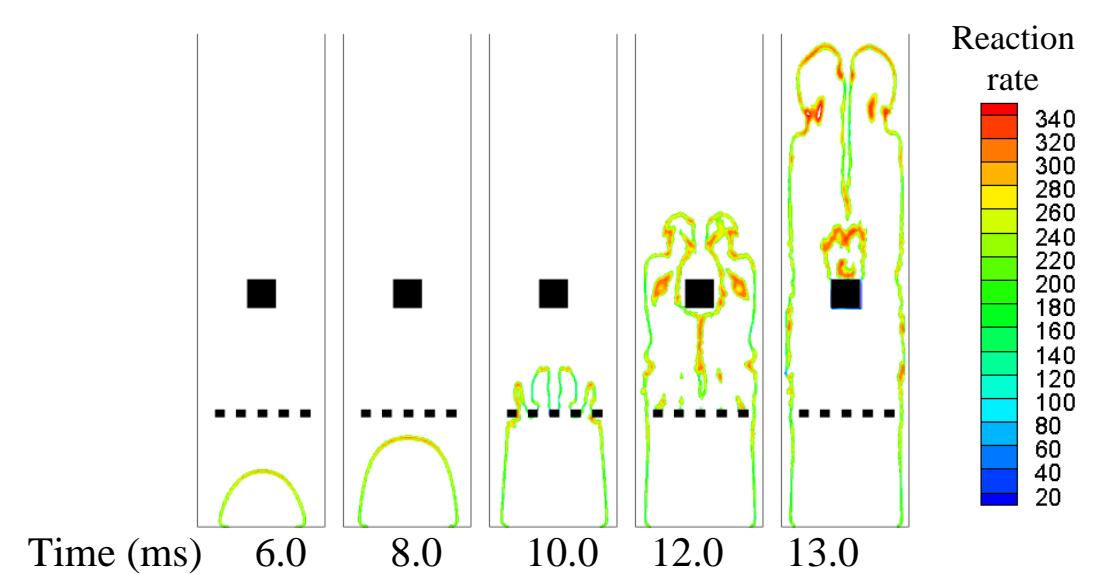

(b) Configuration 6

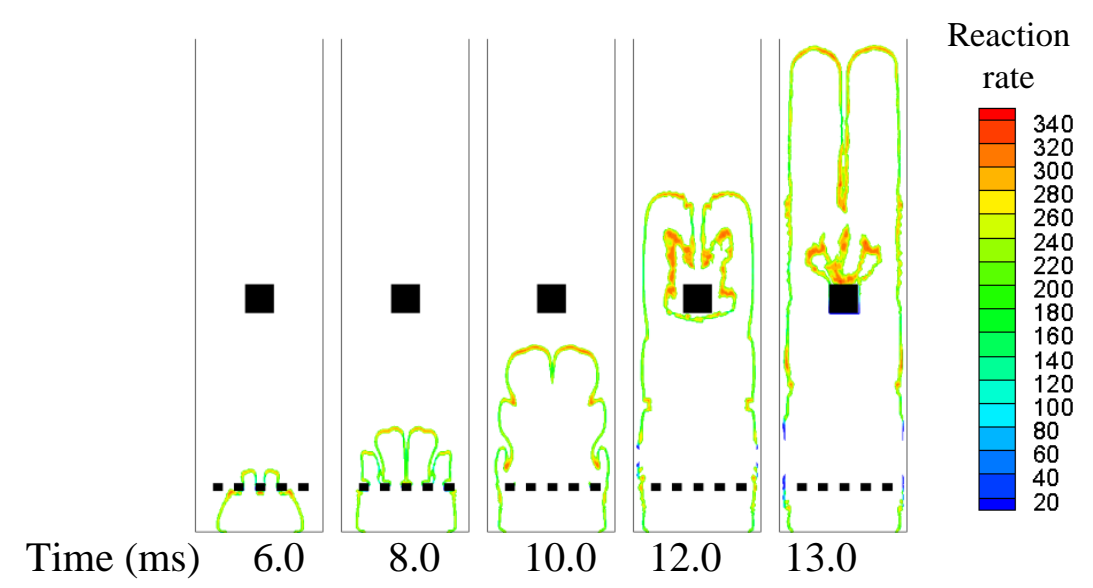

(c) Configuration 7

Figure 16 